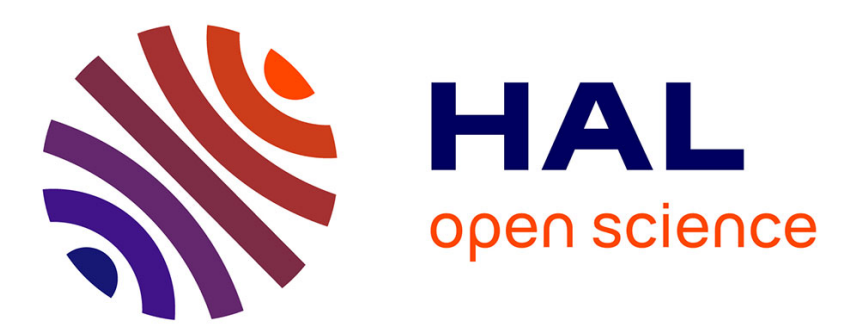

\title{
Arc-elasticity and hierarchical exploration of the neighborhood of solutions in mechanical design
}

Arnaud Collignan, Patrick Sebastian, Jérôme Pailhes, Yann Ledoux

\section{To cite this version:}

Arnaud Collignan, Patrick Sebastian, Jérôme Pailhes, Yann Ledoux. Arc-elasticity and hierarchical exploration of the neighborhood of solutions in mechanical design. Advanced Engineering Informatics, 2012, 26 (3), pp.603-617. 10.1016/j.aei.2012.04.001 . hal-01020838

\section{HAL Id: hal-01020838 \\ https://hal.science/hal-01020838}

Submitted on 8 Jul 2014

HAL is a multi-disciplinary open access archive for the deposit and dissemination of scientific research documents, whether they are published or not. The documents may come from teaching and research institutions in France or abroad, or from public or private research centers.
L'archive ouverte pluridisciplinaire HAL, est destinée au dépôt et à la diffusion de documents scientifiques de niveau recherche, publiés ou non, émanant des établissements d'enseignement et de recherche français ou étrangers, des laboratoires publics ou privés. 


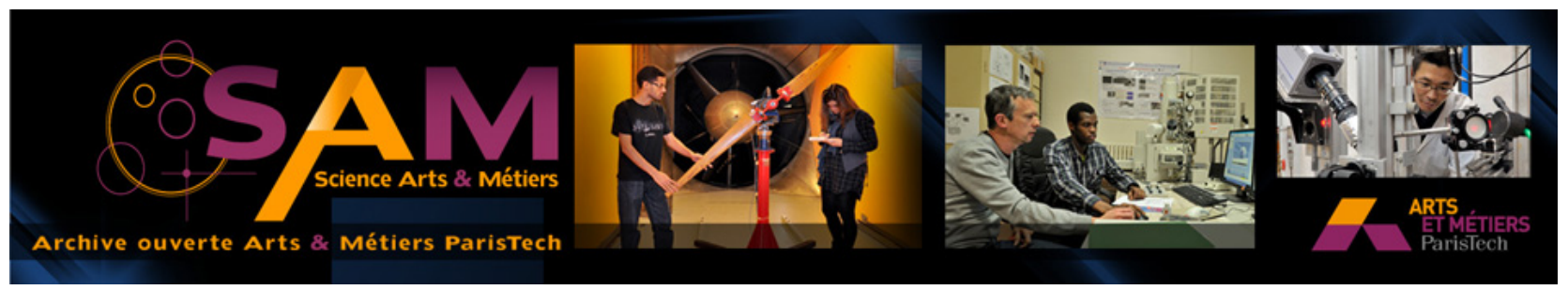

Science Arts \& Métiers (SAM)

is an open access repository that collects the work of Arts et Métiers ParisTech researchers and makes it freely available over the web where possible.

This is an author-deposited version published in: http://sam.ensam.eu

Handle ID: .http://hdl.handle.net/10985/8332

\section{To cite this version :}

Arnaud COLLIGNAN, Patrick SEBASTIAN, Jérôme PAILHES, Yann LEDOUX - Arc-elasticity and hierarchical exploration of the neighborhood of solutions in mechanical design - Advanced Engineering Informatics - Vol. 26, n`3, p.603-617 - 2012 


\title{
Arc-elasticity and hierarchical exploration of the neighborhood of solutions in mechanical design
}

\author{
Arnaud Collignan ${ }^{a}$, Patrick Sebastian ${ }^{\mathrm{a}, *}$, Jérôme Pailhes ${ }^{\mathrm{b}}$, Yann Ledoux ${ }^{\mathrm{a}}$ \\ a Université de Bordeaux, I2M-IMC, Esplanade des Arts E' Métiers, 33405 Talence, Cedex, France \\ ${ }^{\mathrm{b}}$ Arts et Métiers Paristech, I2M-IMC, Esplanade des Arts E' Métiers, 33405 Talence, Cedex, France
}

Keywords:

Optimization

Mechanical design

Arc-elasticity

Design space

Genetic algorithm

\begin{abstract}
A B S T R A C T
In most industrial design processes, the approaches used to obtain a design solution that best fits the specification requirements result in many iterations of the "trial-and-error" type, starting from an initial solution. In this paper, a method is proposed to formalize the decision process in order to automate it, and to provide optimal design solutions. Two types of knowledge are formalized. The first expresses the satisfaction of design objectives, relating to physical behaviors of candidate design solutions. This formalization uses three models, an observation one, an interpretation one and an aggregation one; every design solution is qualified through a single performance variable (a single objective function). The second model is related to modifications that may or may not be applicable to the pre-existing solution. The Designer is often able to define preferences concerning design variables. Some modifications related to this pre-existing solution, can be preferred to other ones. A hierarchy of design variables is proposed to formalize these preferences. The concept of arc-elasticity is introduced as a post-processing indicator to qualify candidate solutions through a trade-off between the performance improvement and their relative distances to the initial solution. The proposed method is used and applied to a riveted assembly, and a genetic algorithm is used to identify optimal solutions.
\end{abstract}

\section{Introduction}

In industrial processes, a typical occurrence in sub-contracting mechanical design, design activity is based on companies' knowhow as well as on designer's imprecise knowledge [1]. These processes require several iterations between product design and simulation, starting from a predefined solution. The aim of this "trial-and-error" approach $[2,3]$ is to obtain a product that corresponds to criteria defined in the design specification documents, via an iterative process of decision-making and optimization. Optimization is mostly based on knowledge rather than on numerical optimization methods. This time-consuming process provides no guarantee of approaching an optimal solution and no justification of the decision process. During such processes, designer preferences linked to the initial solution and to the behavior of the product are applied.

In the following paper, reasoning is based on a single solution, called the reference solution or initial solution since it is involved in an iterative process; this reference solution is related to designer preference and supports the mathematical formulation of this preference. Indeed, design activity is often underpinned by one

\footnotetext{
* Corresponding author. Tel.: +33 (0) 556845 414; fax: +33 (0) 556845436 .

E-mail address: patrick.sebastian@trefle.u-bordeaux.fr (P. Sebastian).
}

pre-existing solution whose structure is regarded as preferable even if it is not precisely adapted to the ongoing design problem. The design and development (prototyping, testing, and industrialization) of this reference solution may require significant investment (costs and delays); and by the end of the process this solution is regarded as well known and secure. The structure of new design solutions is implicitly constrained to remain close to this initial solution as any difference between a candidate solution and the initial solution will imply additional costs [4]. Thus, it is possible to formalize user preferences as a distance between optimal and initial solutions.

The majority of product design optimization problems are regarded as being "multi-objective" [1]: satisfying one of the product's performance criteria, which are related to physical observation variables, is linked to the performance of the other observation variables. Ullman proposes a list of the main elements that must be taken into account in making decisions for this kind of problem: design alternatives and human preferences [5]. Human preference is therefore a major element in design and Augusto et al. [6] distinguishes a priori and a posteriori decision assistance to express these preferences. A priori decision support and designer's preferences concerning the product are formulated in the mathematical optimization problem in the same ways as the physical behavior model of the product. 
Numerous methods can be used to tackle multi-objective problems taking designer's preferences into account. Jones et al. [7] describes three of these methods, namely the reference point method, goal programming and compromise programming. Romero underlines similarities in these approaches. All of these methods are based on a precise modeling of the design objectives [8]. The optimization process then consists in minimizing the gap between the candidate design solutions and these objectives. Other methods use designer's preferences formalized through design decision-making methods [9]. Utility theory [10], introduced in economics, proposes to interpret an observation variable through a utility curve formalizing the preference. Marston illustrates this method [11]. Starting from the Utility theory, Antonsson has developed the Method of Imprecision (MoI) where multiple variables are qualified using fuzzy logic and are aggregated through design strategies $[12,13]$. These aggregation strategies are structured by design axioms to ensure their design-ready property [14]. The Observation-Interpretation-Aggregation method (OIA) is a similar process, structuring the modeling of product behavior and preferences through three models $[15,16]$. The first is a predictive model of the product's behavior; the second qualifies the observation variables using desirability curves [17], while the third aggregates the resulting interpretation variables, formalizing and prioritizing the design objectives via an aggregation step [18,19]. The OIA method is employed to quantify the product's performance through a single variable.

Later on, we consider two types of variables: the product's performance and the distance between one candidate solution and a reference solution. Then, the concept of arc-elasticity is introduced as a decision support indicator of the relative improvements or degradations in these two variables. Elasticity has been introduced in the area of microeconomics [20]; Allen and Lerner [21] proposes the principle of arc-elasticity based on the measurement of the elasticity between two points. In this paper, this concept is applied in engineering design to quantify trade-offs in the selection of different candidate solutions.

From this preference trade-off an original optimization method is proposed. The paper begins by defining the OIA modeling method and the three resulting models. In the second part, the arc-elasticity indicator is presented and adapted to the design field. Next, from this model and this indicator, we propose a global optimization method based on the sequential search for optimal solutions using a hierarchy of design variables. This process is described in the third part of the article. Finally, in the fourth part, the method is illustrated by applying it to a fastened assembly (with rivets) and by optimizing the mechanical system with a genetic algorithm.

\section{Modeling methodology of performance}

Multi-objective optimization problems consist in finding optimal values for every observation variable. Designer's expectations relative to these variables are generally conflicting [19]. Solving multi-objective optimization problems must be performed through a trade-off between the different candidate solutions. This trade-off, derived from an aggregation process, is used to pass from several variables to a single one.

We argue that designer's preferences deriving from his knowhow and experience are not formalized in the observation model. However, designer's preferences are used to interpret every observation variable. Designers have information that enables them to decide whether a value is acceptable or not, information which can be formalized. In the following, preferences are formalized using mathematical functions. The Observation-InterpretationAggregation approach (OIA) is used to translate the design problem into a mono-objective function including both physical behavior and decision models. The OIA approach, detailed in the present section, is divided into three models (Fig. 1):

1. The observation model $\mu$ is a model of the behavior of the product (physics, economics, etc.).

2. The interpretation model $\delta$ expresses the design criteria translating physical observation variables into desirability levels.

3. The aggregation model $\xi$ formalizes and defines priorities between the design objectives.

\subsection{Design variables and observation model}

Design variables (also called decision variables or design parameters according to some authors) are related to the main structural characteristics of the system that must be quantified by the designers and correspond to the degrees of freedom on which designers act to define the system; system performances result from the values of these variables. In the following, $\mathrm{X}$ is defined as the vector containing every design variable $x_{i}$. The design search space $\bar{\Omega}$ (Fig. 1) is defined as the space containing every candidate solution of the optimization problem. Therefore, this space is formed from every possible instantiation of the vector $\mathrm{X}$.

Every design variable $x_{i}$ is associated with a value domain bounding the admissible values of the variable. The value domain of $x_{i}$ is denoted as $\left[\bar{x}_{i}^{-} ; \bar{x}_{i}^{+}\right]$. Therefore the design search space and the design variables vector satisfy:

$$
\begin{aligned}
& \mathrm{X} \in \bar{\Omega} \\
& \text { with } \mathrm{X}=\left[\begin{array}{lllll}
x_{1} & \ldots & x_{i} & \ldots & x_{n}
\end{array}\right]^{T} \\
& \text { and } \bar{\Omega}=\left\{\left[\bar{x}_{1}^{-} ; \bar{x}_{1}^{+}\right] \ldots\left[\bar{x}_{i}^{-} ; \bar{x}_{i}^{+}\right] \cdots\left[\bar{x}_{n}^{-} ; \bar{x}_{n}^{+}\right]\right\}^{T}
\end{aligned}
$$

Value domains can be continuous (dimensions, energy quantities, etc.) or discrete (materials, numbers of parts, product architectures, etc.). Their boundaries are defined by designers, from design requirement documents and also from his expertise.

An instantiated vector $\mathrm{X}$ defines a candidate solution. From this solution, the observation model computes the observation variables $y_{i}$, which forms the vector Y. These variables are used to observe the product's behavior from which the performances of the product are derived. The observation model verifies:

$$
\mu(\mathrm{X})=\mathrm{Y} \text { with } \mathrm{Y}=\left[\begin{array}{lllll}
y_{1} & \ldots & y_{i} & \ldots & y_{m}
\end{array}\right]^{T}
$$

\subsection{Interpretation model}

Observation variables are then translated into an interpretation variable (vector $Z$ ) through the interpretation model. We propose to build formal interpretation functions using desirability functions, but other "value functions" can be employed [22]. The desirability notion was first suggested by Harrington [17] in the area of quality, and is commonly used in multi-objective optimization

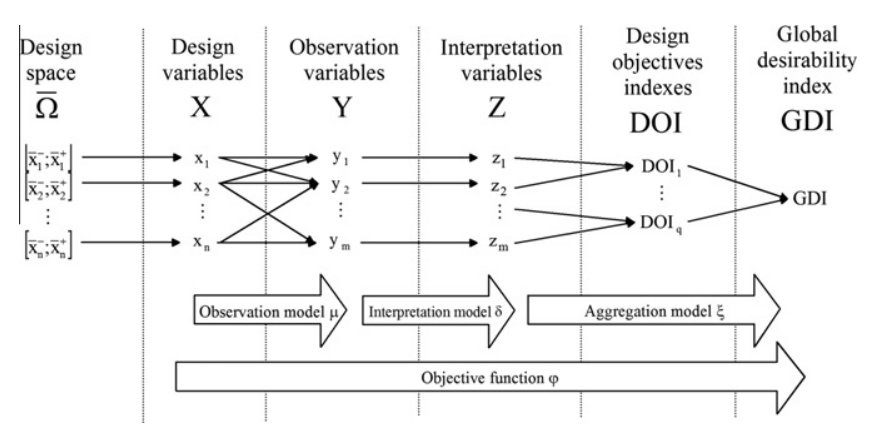

Fig. 1. Modeling methodology. 
problems [23]. Every observation variable $y_{i}$, is associated with a desirability function $d_{i}$ as an input variable and results in a variable $z_{i}$. Interpretation variables are dimensionless values ranging between 0 and 1 (Eq. (3)). The closer an interpretation variable value is to 1 , the more desirable is the value of the corresponding observation variable.

$$
\delta(\mathrm{Y})=\mathrm{Z}=\left[\begin{array}{lllll}
z_{1} & \ldots & z_{i} & \ldots & z_{m}
\end{array}\right]^{T} \text { with } z_{i}=d_{i}\left(y_{i}\right) \text { and } z_{i} \in[0 ; 1]
$$

where $d_{i}$ is a desirability function associated with the observation variable $y_{i}$.

Usually, design constraints are translated into strict constraints using inequality equations. The desirability functions translate design constraints into soft-constraints, which allow a more realistic definition of the design problem. Harrington's desirability functions are continuous functions computed from the coordinates of two points called Accurate Constraint (AC) and Soft Limit (SL) points. These particular points correspond to desirability values of $1 \%$ and $99 \%$ and shade the transition domain of acceptability of the constraint. Desirability functions used in this paper are defined in the Appendix A.4.

\subsection{Aggregation model}

Interpretation variables are finally aggregated according to the design objectives into Design Objective Indexes (DOI), formalizing the satisfaction levels of the objectives. These objectives have been identified through the investigation of the product lifecycle, the product's functions and customer requirements. The DOI are then aggregated into a single mono-objective value called Global Desirability Index (GDI), which qualifies the performance of the candidate solution. This approach aims at simulating common human reasoning: observation of a phenomenon, interpretation of this observation, and global appreciation of the design solution. Classically, design optimization problems are modeled as multi-objective optimization problems [24,25] by combining goals (values to be maximized or minimized) and constraints (equality equations or inequality relations). The OIA method results in a mono-objective optimization problem since goals and constraints are integrated in the same model.

Several types of aggregation are compared by Kros and Mastrangelo [24] including additive aggregation and multiplicative aggregation. Additive aggregation, which is a weighted arithmetic mean, does not satisfy the principle of annihilation, as the aggregated value of a solution can be positive whereas one of the interpretation variable values is null. On the contrary, multiplicative aggregation does satisfy this principle [19], which is one of the main axioms of design-ready aggregation models [14,26]. Consequently, in the following we propose to use a two-step multiplicative aggregation.

Design objectives are related to several interpretation variables. For this reason, we propose to aggregate them into Design Objective Indexes (DOI). These variables quantify the satisfaction level of the solution in relation to every design objective (Fig. 1). For example, a design objective related to product transportability is linked to satisfaction levels (interpretation variables) derived from physical observation variables such as the dimensions and mass of the product. Consequently, in contrast to Derringer [18] and Derringer and Suich [19] who aggregate interpretation variables into a single value, we introduce intermediate steps between the interpretation variables and their aggregation into a single performance variable. Interpretation variables are aggregated as follows:

$\mathrm{DOI}_{i}=\prod_{j=1}^{p_{i}}\left[\left(z_{j}\right)^{\omega_{j}}\right]$ with $\omega_{j} \geqslant 0$ and $\sum_{j=1}^{p_{i}} \omega_{j}=1$ where $\mathrm{DOI}_{i}$ is the $i$ th Design Objective Index, $p_{i}$ is the number of aggregated interpretation variables in the $\mathrm{DOI}_{i}$ and $\omega_{j}$ is a weighting parameter. Weighting parameters are set to balance priorities between the interpretation variables. The higher is the value of the weighting parameter $\omega_{j}$, the higher is the relative priority of the corresponding interpretation variable $z_{j}$.

In the aggregation model, interpretation variables must be employed at least once and one interpretation variable can contribute to the definition of several different DOI indexes. From a practical point of view, weighting parameters $\omega_{j}$ result from a trade-off between experts linked to several design objectives.

Once the different DOI indexes have been calculated, they are then aggregated into the global desirability index GDI (Eq. (5))

$\mathrm{GDI}=\prod_{j=1}^{q}\left[\mathrm{DOI}_{i}^{v_{j}}\right]$ with $v_{j} \geqslant 0$ and $\sum_{j=1}^{q} v_{j}=1$

where $q$ is the number of DOIs and $v_{j}$ is a weighting parameter associated with $\mathrm{DOI}_{j}$.

The entire OAI approach can be represented mathematically by the objective function $\varphi$ described in Eq. (6).

$\varphi(\mathrm{X})=\xi \circ \delta \circ \mu(\mathrm{X})=G D I$

where "o" is a function composition. (7)):

The optimization problem can thus be expressed as follows (Eq.

find $\mathrm{X}^{*} / \mathrm{X} \in \bar{\Omega}, \max (\varphi(\mathrm{X}))$

where solution $\mathrm{X}^{*}$ is the optimal solution.

\section{Arc-elasticity}

The previous paragraph details the OIA approach, which results in a performance objective function. In this paragraph, we consider another global objective related to an initial reference design solution. The notion of arc-elasticity is introduced, and is defined as a ratio between gain of performance and detachment from the initial solution. Since the initial solution is regarded as being preferable, arc-elasticity is used as a trade-off indicator.

\subsection{Notion of arc-elasticity}

The concept of elasticity was first introduced in 1890 [21] in microeconomics to quantify a product demand variation $(Q)$ according to a price variation $(P)$ in a particular situation.

Although it has its origin in economics, the concept of elasticity has been used in studies covering a wide variety of fields. Sankarasubramanian used it in climatology to produce a map of the USA showing elasticity in river currents in relation to rainfalls [27]. When modeling infection in a salmon population, Fenichel used elasticity to point out the sensitivity of many parameters in the model [28]. Lastly, Rassafi suggested an aggregation method with several elasticities to produce an eco-criterion in the field of freight and passenger transport [29].

Considering two distinct situations and when the relation between $\mathrm{P}$ and $\mathrm{Q}$ is not differentiable, another expression of elasticity $\varepsilon$ can then be used, called "arc-elasticity" $[21,30]$.

Let us consider two situations $\mathrm{S}^{1}$ and $\mathrm{S}^{2}: \mathrm{P}^{1}$ (resp. $\mathrm{P}^{2}$ ) is the price and $\mathrm{Q}^{1}$ (resp. $\mathrm{Q}^{2}$ ) is the demand in the situation $\mathrm{S}^{1}$ (resp. $\mathrm{S}^{2}$ ). Arc-elasticity $\varepsilon$ (see also Fig. 2) is defined by the following equation: 

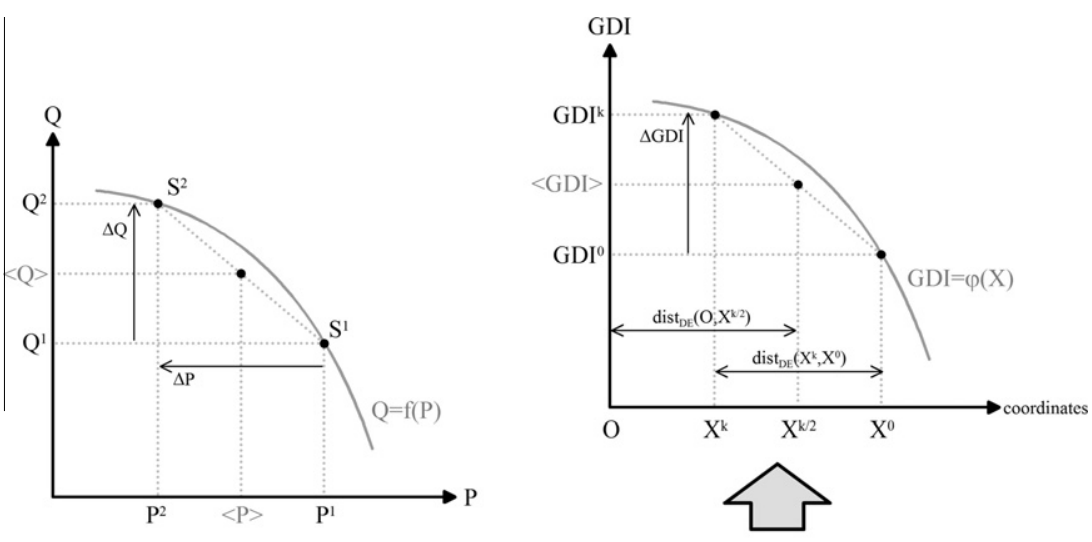

1. Arc-elasticity definition

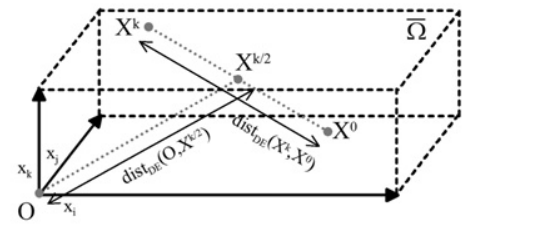

2. Arc-elasticity in design

Fig. 2. Schema for the arc-elasticity notion.

$\varepsilon=\frac{\Delta \mathrm{Q} \%}{\Delta \mathrm{P} \%}=\frac{\left(\frac{\Delta \mathrm{Q}}{\langle\mathrm{Q}\rangle}\right)}{\left(\frac{\Delta \mathrm{P}}{\langle\mathrm{P}\rangle}\right)}=\frac{\left(\frac{\mathrm{Q}^{2}-\mathrm{Q}^{1}}{\frac{1}{2}\left(\mathrm{Q}^{2}+\mathrm{Q}^{1}\right)}\right)}{\left(\frac{\mathrm{P}^{2}-\mathrm{P}^{1}}{\frac{1}{2}\left(\mathrm{P}^{2}+\mathrm{P}^{1}\right)}\right)}$

with $\Delta \mathrm{Q} \%=\frac{\Delta \mathrm{Q}}{\langle\mathrm{Q}\rangle}$ and $\left\{\begin{array}{c}\Delta \mathrm{Q}=\mathrm{Q}^{2}-\mathrm{Q}^{1} \\ \langle\mathrm{Q}\rangle=\frac{1}{2}\left(\mathrm{Q}^{2}+\mathrm{Q}^{1}\right)\end{array}\right.$ (resp. $\left.\mathrm{P}\right)$

Allen introduced three properties related to the definition of arcelasticity [21]:

1. Independence of the units: Arc-elasticity has an intrinsic meaning, as the percentage variation of $\Delta \mathrm{Q} \%$ for a $1 \%$ change of $\Delta \mathrm{P} \%$.

2. Symmetry with respect to the two situations: Both are of equal importance when calculating $\varepsilon$.

3. Equality to the unity, if the two situations are equivalent: $\varepsilon=1$ indicates that the variations of $\mathrm{Q}$ and $\mathrm{P}$ are totally equivalent in the two situations.

In this paper, arc-elasticity is applied in design by considering an initial solution and a candidate solution. Arc-elasticity is regarded as a numerical value suitable for quantifying a trade-off between performance and "overall cost" of the modification of an initial reference solution. It is noticeable that in this paper two distinct concepts of cost are taken into account:

- The overall cost of the transition between an initial solution and an optimal solution [4]. This refers to monetary costs but also to cost in terms of time, psychological stress or risk.

- The unit cost of a candidate solution, independent of the initial solution and only depending on costs of materials, parts, machining, etc. This unit cost is employed in the Section 5.

In the present case, arc-elasticity is used to qualify the relevance of design decisions made during the design of the initial solution and, if need be, to propose optimal solutions.

"Cost is in fact what is removed as a result of action on available resources (...) the individual acts when the benefit (...) he derives from the action is noticeably greater than the internal resources that have to be devoted to it."
This extract from Alexandre and Gasparski [4] illustrates the notion of arc-elasticity proposed in this paper, where the "benefit" corresponds to an improvement in performance compared to the initial solution and the "cost" is a distance between initial and candidate solutions. The initial solution is written $\mathrm{X}^{0}$, and its components $x_{i}^{0}$.

Let us consider two solutions: the initial one with performance $\mathrm{GDI}^{0}$ and a candidate solution $\mathrm{X}^{k}$ with performance $\mathrm{GDI}^{k}$. As a trade-off indicator, the arc-elasticity between these two solutions takes into account the variation of the performance of $X^{k}$ in relation to $\mathrm{X}^{0}: \Delta \mathrm{GDI}=\mathrm{GDI}^{k}-\mathrm{GDI}^{0}$. This variation corresponds to the variable $\mathrm{Q}$ in Eq. (8).

The variation of $\mathrm{P}$ corresponds to the overall cost, which is assimilated to the distance between the two solutions in the design space. The arc-elasticity of the performance is thus defined as the quotient of the dimensionless variation in performance $\Delta \mathrm{GDI}$ and the dimensionless distance between $\mathrm{X}$ and $\mathrm{X}^{0}$.

\subsection{Distance between solutions}

Many definitions of distance can be found, which are adapted to different cases of application. The most frequently used of these definitions is the Euclidean distance:

$\operatorname{dist}_{E}\left(\mathrm{X}^{1}, \mathrm{X}^{2}\right)=\sqrt{\sum_{i=1}^{n}\left(x_{i}^{2}-x_{i}^{1}\right)^{2}}$

where $\mathrm{X}^{1}$ (with component $x_{i}^{1}$ ) and $\mathrm{X}^{2}$ (with component $x_{i}^{2}$ ) are two solutions in the design space $\bar{\Omega}$.

Unlike Augusto, who calculates a distance between two candidate solutions by taking into account design variables and observation variables in the same equation [6], we propose to apply the Euclidean distance since the observation variables are already integrated into the arc-elasticity expression through $\Delta \mathrm{GDI}$.

Design variable $x_{i}$ may correspond to different physical units. Therefore, it is necessary to transform $x_{i}$ into a non-dimensional value ranging between 0 and 1 from the values of $\bar{X}_{i}^{-}$and $\bar{X}_{i}^{+}$. Eq. (10) expresses this dimensionless Euclidean distance: 


$$
\begin{aligned}
\operatorname{dist}_{\mathrm{DE}}\left(\mathrm{X}^{1}, \mathrm{X}^{2}\right) & =\sqrt{\sum_{i=1}^{n}\left(\left[\frac{x_{i}^{2}-\bar{x}_{i}^{-}}{\bar{x}_{i}^{+}-\bar{x}_{i}^{-}}\right]-\left[\frac{x_{i}^{1}-\bar{x}_{i}^{-}}{\bar{x}_{i}^{+}-\bar{x}_{i}^{-}}\right]\right)^{2}} \\
& =\sqrt{\sum_{i=1}^{n}\left(\frac{x_{i}^{2}-\bar{x}_{i}^{1}}{\bar{x}_{i}^{+}-\bar{x}_{i}^{-}}\right)^{2}}
\end{aligned}
$$

\subsection{Taking into account performance and distance}

To qualify a candidate design solution $\mathrm{X}$, two variables have to be computed: the performance (GDI) which has to be as high as possible, and the dimensionless Euclidean distance between $\mathrm{X}$ and $\mathrm{X}^{0}\left(\right.$ dist $\left._{\mathrm{DE}}\right)$ which has to be as low as possible. Fig. 3 illustrates a set of candidate solutions from the application case proposed in the last part of this paper. Each point (black cross) corresponds to a particular design solution and shows the GDI and dist ${ }_{D E}$ values of the solutions.

The Pareto frontier (through grey squares) is illustrated in Fig. 3, which constitutes the set of non-dominated solutions regarding GDI and dist $t_{\mathrm{DE}}$. The two ultimate solutions of the Pareto frontier are:

- The initial solution $X^{0}$ (black circle) which has a zero distance to itself.

- The solution of highest performance, or "GDI-optimal solution" (black square) which has the best GDI value.

As a trade-off indicator, arc-elasticity is used to select solutions from the Pareto frontier [31]. By aggregating GDI and dist ${ }_{D E}$
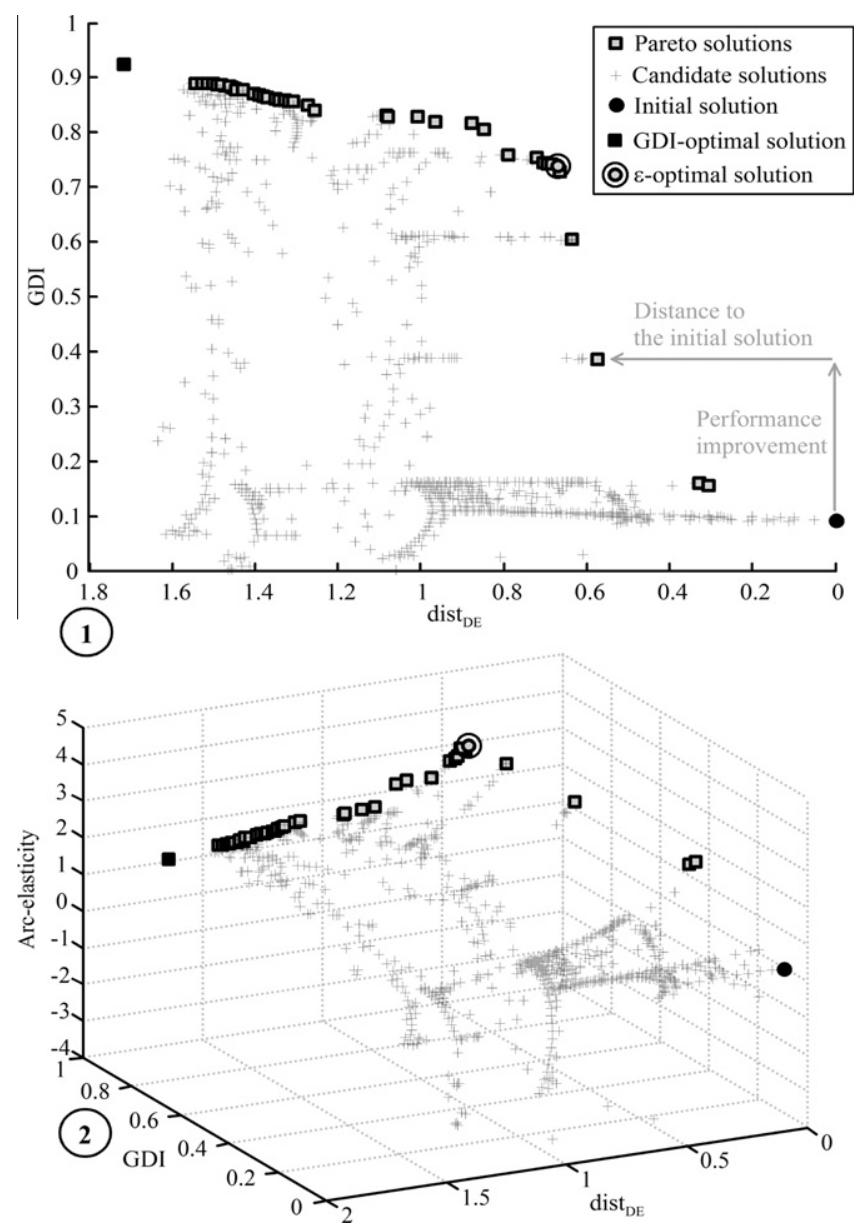

Fig. 3. Pareto frontier and arc-elasticity.
(Eq. (11)), the arc-elasticity returns a unique value $\varepsilon$ used to rank the candidate solutions. Fig. 3 proposes the same graph than the Fig. 3, adding a third dimension corresponding to the arc-elasticity value. The arc-elasticity allows selecting a candidate solution (double grey circle) near the convex Pareto frontier, and close to the initial solution [32]. Arc-elasticity optimization results in optimizing the ratio between the improvement of the performance and the closeness to the initial solution and confirms the interest of this notion in design field. This point has been discussed by Sebastian et al. [32].

\subsection{Arc-elasticity in design}

The definition of the Euclidean distance (Eq. (10)) enables to express arc-elasticity in design:

$\varepsilon=\frac{\left(\frac{\mathrm{GDI}^{k}-\mathrm{GDI}^{0}}{\frac{1}{2}\left(\mathrm{GDI}^{k}+\mathrm{GDI}^{0}\right)}\right)}{\left(\frac{\operatorname{dist}_{\mathrm{DE}}\left(\mathrm{X}^{k}, \mathrm{X}^{0}\right)}{\operatorname{dist}_{\mathrm{DE}}\left(0, \mathrm{X}^{k / 2}\right)}\right)}$

where $\mathrm{X}^{0}$ is the initial reference solution, $\mathrm{X}^{k}$ is the candidate solution, $O$ is the origin of the design space and $X^{k / 2}$ is the middle of the segment $\left[\mathrm{X}^{k} \mathrm{X}^{0}\right]$. It is noticeable that the coordinates of point $\mathrm{O}$ are null.

Fig. 2 illustrates the arc-elasticity concept in the design field. In this figure, axis P (Fig. 2) is replaced by the entire design space $\bar{\Omega}$ (Fig. 2) to illustrate the notion of distance.

The dimensionless Euclidean distance can be projected onto a single axis, e.g. according to a given design variable. In this case, the distance is equivalent to a dimensionless Manhattan distance [33]. This distance relates directly the definition proposed in this paper to the definition of arc-elasticity suggested by Allen; it is the ratio of dimensionless variation of performance and dimensionless variation of the design variable values.

As illustrated on Fig. 4, equivalence is supposed between performance (GDI) and dimensionless Euclidean distance (dist $t_{\mathrm{DE}}$ ). An ideal solution $(\mathrm{GDI}=1)$ is considered as preferable as the initial solution $\mathrm{X}^{0}\left(\operatorname{dist}_{\mathrm{DE}}\left(\mathrm{X}^{0}, \mathrm{X}^{0}\right)=0\right)$. An unacceptable solution $(\mathrm{GDI}=0)$ is as unwanted as a distant candidate solution $\left(\operatorname{dist}_{D E}\left(X, X^{0}\right) \geqslant 1\right.$ ). A candidate solution is considered "distant" if its distance to $\mathrm{X}^{0}$ is higher or equal to the unit. This unit corresponds to the dimensionless length of a value domain.

Fig. 5 maps arc-elasticity $\varepsilon$ as a function of GDI (performance) and dist $_{\mathrm{DE}}$ (overall cost). The arc-elasticity of the initial solution $\mathrm{X}^{0}$ is theoretically infinite or indeterminate. However, this singular configuration of arc-elasticity has no practical interest and, consequently, we propose to fix the arc-elasticity of $\mathrm{X}^{0}$ at zero. The figure

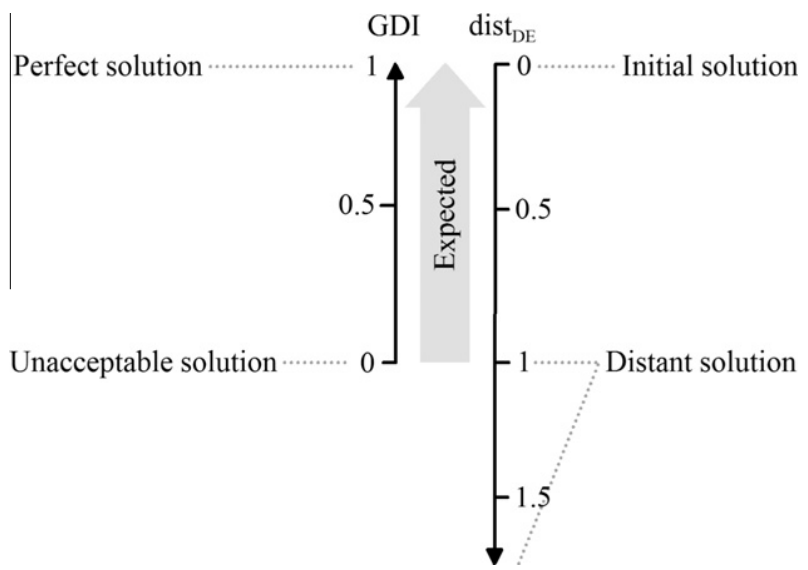

Fig. 4. Equivalence performance/distance. 

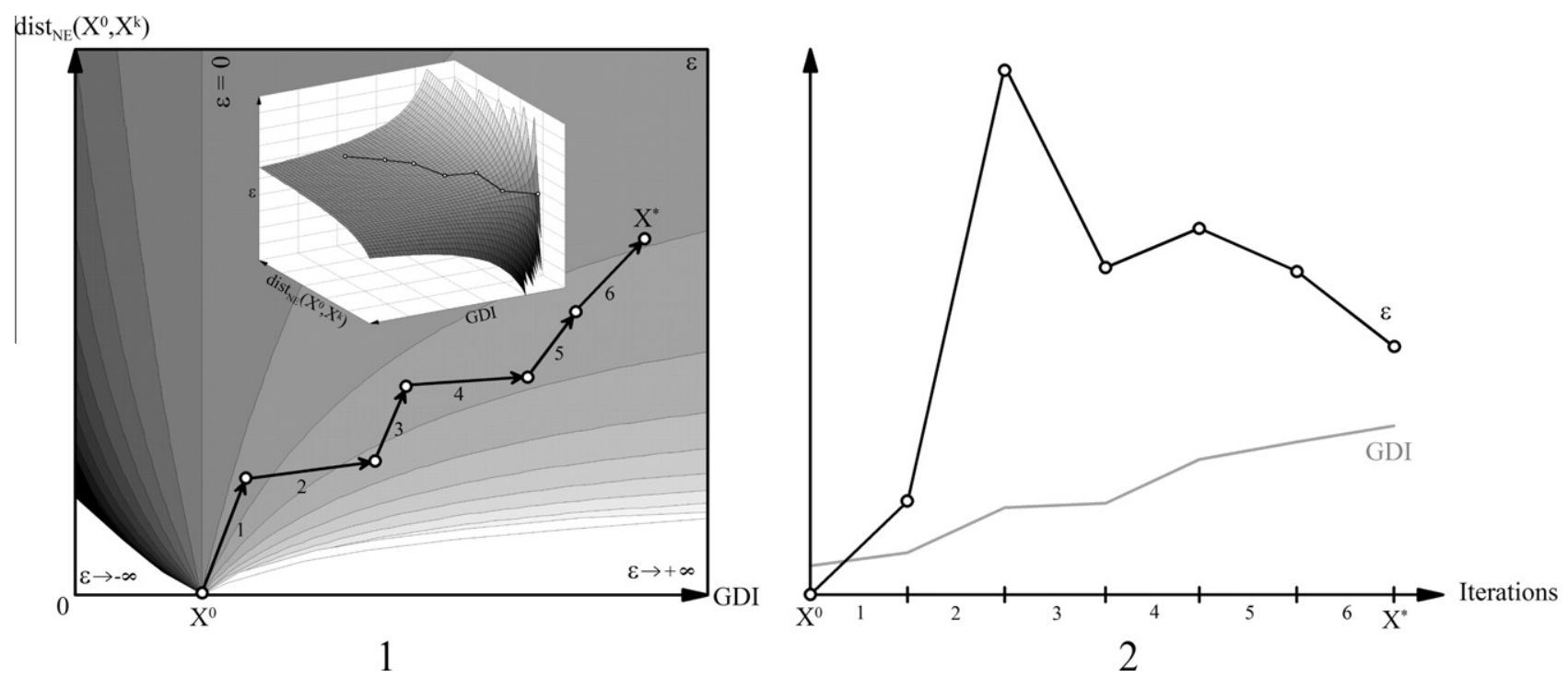

Fig. 5. Moving along the arc-elasticity mapping.

shows that the arc-elasticity value tends towards infinity if the overall cost is low regarding the high performance of the solution; if the performance of a candidate solution is lower than the initial one, then arc-elasticity tends towards minus infinity.

In this paper, we propose to study arc-elasticity as an indicator. The GDI has to be maximized through an iterative optimization process and the variations of $\varepsilon$ are observed. Fig. 5 illustrates an optimization process showing the path from the initial solution to the optimal one. It can be seen that GDI is maximized but $\varepsilon$ can increase or decrease (Fig. 5), depending on the position of the candidate solutions on the arc-elasticity map (Fig. 5).

\section{Exploration of the design space}

The initial reference solution contains a great deal of nonformalized information, e.g. prior knowledge of the problem or non-formal design constraints such as, for instance, preference for certain materials due to supplier or stock constraints. The designer must however transform this reference solution to adapt it to new design problems or improve its performances. In the following, this is done by proposing new values for the design variables, regarded as a modification of the initial solution. Design variable modifications are related to preferences since they may be difficult to perform.

Design modifications are organized into a hierarchy according to these preferences, and from this hierarchy, the optimization process is sequenced by first proposing modifications related to the most preferable transformations defined by the designer. This sequential optimization process is an exploration process since it consists in a sequential exploration of every design variable domain.

Fig. 6 illustrates this exploration process starting from the initial solution:

- The algorithm sequentially considers every design variable, and searches for an optimal solution by modifying the variables according to the hierarchy specified by the designer (cf. $\S 4.1$ ).

- By combining an exploration function and a membership function, every value domain is progressively explored. A design variable is processed when its value domain is modified. At the beginning of the design variable processing, the

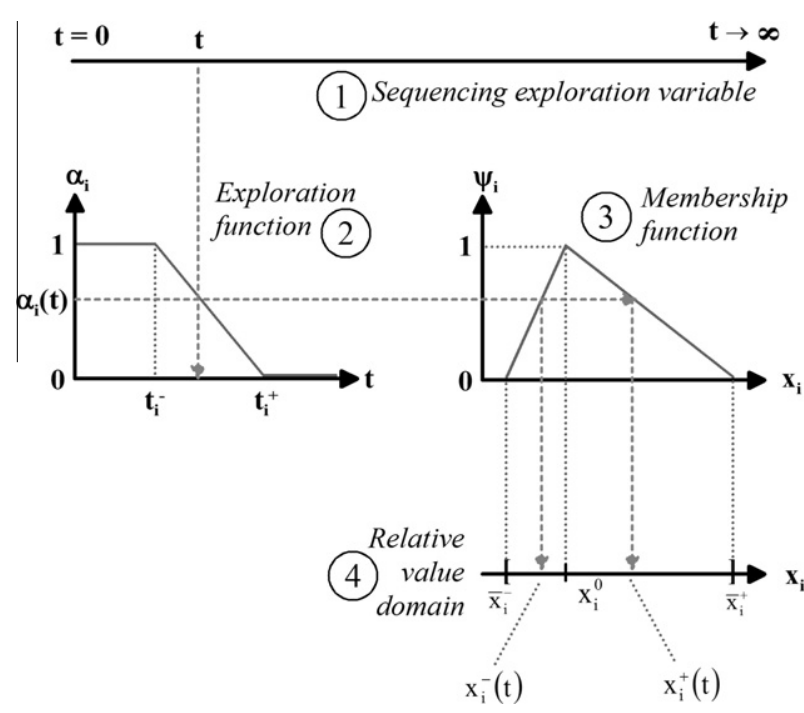

Fig. 6. Progressive exploration of the design space (for a variable $x_{i}$ ).

domain is reduced to a single value. By the end of this process, the algorithm covers the entire corresponding value domain (cf. $\S 4.2$ and 4.3).

- Global optimization algorithms explore the entire design space $\bar{\Omega}$ to find optimal solutions. In the present case, the proposed method is based on the progressive extension of $\bar{\Omega}$ around the initial reference solution $\mathrm{X}^{0}$ and according to the hierarchy of design variables. We use a genetic algorithm as a global optimization algorithm (cf. § 4.4).

\subsection{Hierarchy and sequencing exploration}

The sequencing exploration variable is denoted as $t$, with values ranging from $[0 ;+\infty]$ (Fig. 6 ). During the optimization process, the value of $t$ increases and thus the processing of every design variable can be quantified; every value of $t$ is linked to a corresponding design variable value in the hierarchy. Every value of $t$ authorizes or prohibits the complete or partial exploration of the value domains of every design variable. In an iterative optimization 
algorithm, $t$ can be the current number of iteration during the algorithm's run.

To carry out this process, the notion of relative design space $\Omega(t)$ is proposed and defined as follows:

$$
\begin{aligned}
& \Omega(t) \subset \bar{\Omega} \\
& \text { with } \Omega(t)=\left[\left[x_{1}^{-}(t) ; x_{1}^{+}(t)\right] \ldots\left[x_{i}^{-}(t) ; x_{i}^{+}(t)\right] \ldots\left[x_{n}^{-}(t) ; x_{n}^{+}(t)\right]\right]^{T} \\
& \Longleftrightarrow i \in[1 ; n],\left[x_{i}^{-}(t) ; x_{i}^{+}(t)\right] \subset\left[\bar{x}_{i}^{-} ; \bar{x}_{i}^{+}\right]
\end{aligned}
$$

$\Omega(t)$ is the design space relative to $t$, included in $\bar{\Omega}$ and containing $\mathrm{X}^{0}$.

Thus, depending on the value of $t$, the temporary domain of values of every design variable $x_{i}$ is denoted as $\left[\bar{x}_{i}^{-}(t) ; \bar{x}_{i}^{+}(t)\right]$. According to the value of $t$, this temporary domain satisfies the following:

- If the design variable has already been processed: $\left[\bar{x}_{i}^{-}(t) ; \bar{x}_{i}^{+}(t)\right]=\left[\bar{x}_{i}^{-} ; \bar{x}_{i}^{+}\right]$.

- If the design variable has not yet been processed: $\left[\bar{x}_{i}^{-}(t) ; \bar{x}_{i}^{+}(t)\right]=\left\{x_{i}^{0}\right\}$.

- If the design variable is being processed the temporary domain depends on the values of $t$ and the parameters of the exploration function.

\subsection{Exploration functions}

In order to link the sequencing exploration variable $t$ to the design variables, we propose to introduce the notion of exploration functions. For every design variable $x_{i}$ this function is denoted as $\alpha_{i}(t)$ and is defined as follows:

- If $\alpha_{i}(t)=1$, the value domain of the design variable has not been processed,

- If $\alpha_{i}(t)=0$ the entire value domain is explored,

- If $1>\alpha_{i}(t)>0$ some compact part of the value domain $\left[\bar{x}_{i}^{-} ; \bar{x}_{i}^{+}\right]$is explored.

For the design variable $x_{i}$, the exploration function $\alpha_{i}(t)$ is a decreasing linear piecewise function (Fig. 6) satisfying to:

$$
\alpha_{i}(t)=\left\{\begin{array}{cc}
1, & \text { for } t<t_{i}^{-} \\
\frac{t_{i}^{+}-t}{t_{i}^{+}-t_{i}^{-}}, & \text {for } t_{i}^{-} \leqslant t \leqslant t_{i}^{+} \\
0, & \text { for } t_{i}^{+}<t
\end{array}\right.
$$

where $t_{i}^{-}$and $t_{i}^{+}$are two parameters of the function $\alpha_{i}$ defining its decrease rate between 1 and 0 .

The pairs of parameters $\left(t_{i}^{-} ; t_{i}^{+}\right)$define the ranks of the design variables in the hierarchy. For example, let us consider two design variables $x_{i}$ and $x_{j}$ with $t_{i}^{+}<t_{j}^{-}$. This inequality relation means that $x_{i}$ is prior to $x_{j}$ in the hierarchy, i.e. the designer wants to process $x_{i}$ completely before starting to process $x_{j}$ (Fig. 7).

Within the framework of the application presented in the following paragraphs and as shown in Fig. 7, it is proposed to use exploration functions, distributed uniformly along axis $t$. A fixed parameter $p$ is introduced which defines the slope of every function $\alpha_{i}$ as the difference between its parameters $t_{i}^{-}$and $t_{i}^{+}$. The parameter $p$ is also defined as the value separating the exploration of the value domains of two consecutive design variables (Fig. 7). Thus, for two design variables $\mathrm{x}_{\mathrm{i}}$ and $\mathrm{x}_{\mathrm{j}}$, in successive positions in the hierarchy: $p=t_{j}^{-}-t_{i}^{+}$. The expression of the parameters of every exploration function is generalized as shown in Eq. (14):

$\forall x_{i},\left\{\begin{array}{c}t_{i}^{-}=\left(2 . \operatorname{order}_{i}-1\right) p \\ t_{i}^{+}=2 \text { order }_{i} \cdot p\end{array}\right.$

where $\operatorname{order}_{i}$ corresponds to the place of $x_{i}$ in the design variable hierarchy.
For example, for the riveted junction presented in $\S 5.1$ the hierarchy is defined in Table 2 according to the difficulty inherent in modifying the different design variables.

\subsection{Boundaries of the value domains}

In this paragraph, the method used to modify the boundaries of the relative value domains is detailed. Design variables are associated to triangular membership functions. For every design variable $x_{i}$, the corresponding membership function $\psi_{i}\left(x_{i}\right)$ has a value of:

- 1 if the value of the variable is identical to that of the initial solution $\left(x_{i}=x_{i}^{0}\right)$.

- 0 if the value of the variable is one of the boundaries of its value domain $\left(x_{i}=\bar{x}_{i}^{-}\right.$or $\left.x_{i}=\bar{x}_{i}^{+}\right)$.

The triangular membership function $\psi_{i}$ is defined as follows:

$\psi_{i}\left(x_{i}\right)=\left\{\begin{array}{cc}0, & \text { if } x_{i} \leqslant ; \bar{x}_{i}^{-} \\ x_{i}\left(x_{i}^{0}-\bar{x}_{i}^{-}\right)+\bar{x}_{i}^{-}, & \text {if } ; \bar{x}_{i}^{-}<x_{i}<x_{i}^{0} \\ 1, & \text { if } x_{i}=x_{i}^{0} \\ x_{i}\left(x_{i}^{0}-\bar{x}_{i}^{+}\right)+\bar{x}_{i}^{+}, & \text {if } x_{i}^{0}<x_{i}<; \bar{x}_{i}^{+} \\ 0, & \text { if } x_{i} \geqslant ; \bar{x}_{i}^{+}\end{array}\right.$

For a given value of $t$, a cut is made on every membership function (Fig. 6) to determine the boundaries of the temporary value domains. These boundaries satisfy the following equation:

$$
\begin{aligned}
& \text { Find }\left(x_{i}^{-}(t) ; x_{i}^{+}(t)\right) \in\left[\bar{x}_{i}^{-} ; \bar{x}_{i}^{+}\right]^{2} \\
& \quad \text { with } \alpha_{i}(t)=\psi_{i}\left(x_{i}^{-}(t)\right)=\psi_{i}\left(x_{i}^{+}(t)\right) \\
& \quad \text { and } x_{i}^{-}(t) \leqslant x_{i}^{+}(t)
\end{aligned}
$$

Thus, if the value of the exploration function $\alpha_{i}(t)$ equals 1 , the value domain of $x_{i}$ is limited to the value of $x_{i}^{0}$. If $\alpha_{i}(t)$ equals zero, then the temporary value domain of $x_{i}$ matches the domain $\left[\bar{x}_{i}^{-} ; \bar{x}_{i}^{+}\right]$.

Fig. 6 summarizes the exploration process for the design variable $x_{i}$. Using the sequencing exploration of variable $t$ (Fig. 6) the cut on $x_{i}$ is defined via its exploration function $\alpha_{i}$ (Fig. 6). For any value of $t$ and every design variable $x_{i}$, the cut defines the relative value domain that bounds the exploration and guides the optimization process (Fig. 6). Therefore, for every design variable $\mathrm{x}_{\mathrm{i}}$, this method requires a definition for the following:

- A value domain $\left[\bar{x}_{i}^{-} ; \bar{x}_{i}^{+}\right]$.

- An initial value $x_{i}^{0}$.

- A membership function $\psi_{i}$.

- An exploration function $\alpha_{i}$.

\subsection{Genetic algorithm}

The optimization algorithm must perform a global search for design solutions inside the set $\Omega(t)$, and consequently, must be a global optimization algorithm. Many optimization algorithms [34] can be used to make such a global optimization and avoid the traditional "trial-and-error" approach.

In the context of the illustration used in this paper, we use a Genetic Algorithm introduced by Goldberg [35] and developed for instance by Javadi et al. [36]. This particular algorithm (Fig. 8) proves to be robust, polyvalent, and able to process a large number of design variables. It is noticeable that, in an industrial context, this algorithm is already used for numerous applications. The main difficulty in developing the method concerns the modeling process of the OIA method. Such a development may require significant investment in terms of time and financial resources. However, this cost can be offset since the decision models are reusable and may lead to significant performance improvements. Models of 


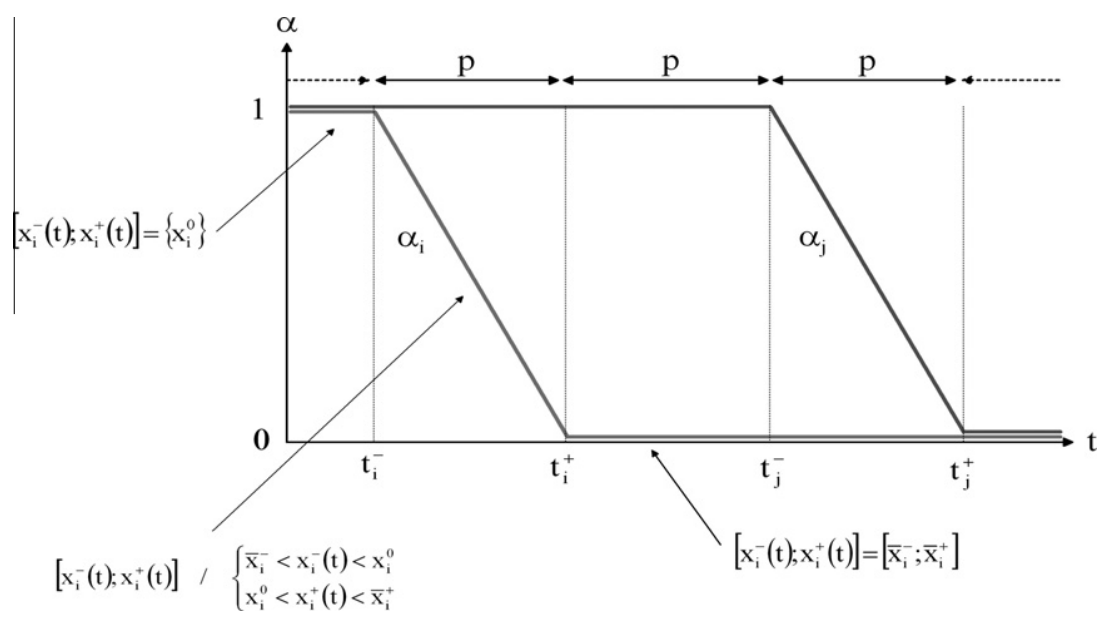

Fig. 7. Exploration functions.

preference formalized through the OIA method are capitalized and reused. The proposed method is applied to problems that:

- Are recurrent.

- Hold up the production process.

- Are time-consuming.

The principle of Genetic Algorithms is based on an analogy between design solution optimization, Darwin's competitive evolution and basic principles of genetics. A population of individuals evolves in a simulated competitive environment. Every individual is regarded as a candidate solution to the design optimization problem. The genes of the individuals correspond to design variables. The genetic algorithm evaluates the performance of every individual through the objective function $\varphi$ (Eq. (6)), selects the best individuals, and applies several genetic operators to simulate natural cross-over and mutation of genes. From these basic operations, new candidate solutions are generated through an iterative process and the algorithm converges towards a global solution satisfying the objectives and constraints of the design problem. In the context of Genetic Algorithms, iterations are called generations.

The gene coding proposed in this paper does not differentiate the phenotype and the genotype of the individuals. In practice, genes correspond directly to design variables and are real values in the genotype representation.

Four genetic operators are used:

- The "selection" operator generates a new population of individuals, selecting solutions with a probability depending on their ranks (the rank is established through the GDI value).

- The "cross-over" operator browses the new population, considering couple of individuals. For each couple, a random number between 0 and 1 is generated; if it reaches the cross-over probability (which is a parameter of the algorithm, see Table 1) the cross-over process is activated: two new individuals are randomly created using the genotype of the two previous selected solutions. Two cuts are realized in the genes to generate the new individuals, which replace the two previous one in the new population of individuals.

- The "mutation" operator browses all the individuals of the new population. For every individual, if a random number reaches the mutation probability (see Table 1) a gene is randomly selected and randomly modified in its corresponding relative values domain.

- The "hill-climbing" operator is similar to the mutation one with a different activation probability (see Table 1), but the selected gene is not randomly modified: its new value is selected in the neighborhood of the previous one. This operator is used to realize a local search in the relative design space.

The stopping criterion for the algorithm is activated as soon as:

- An individual reaches or exceeds a performance limit: $\mathrm{GDI}>\mathrm{GDI}^{\text {stop; }}$

- The number of generations already carried out $\left(\mathrm{N}_{\text {gen }}\right)$ reaches a maximum: $\mathrm{N}_{\text {gen }}>\mathrm{N}_{\text {genMax }}$.

In the next section, the following parameters are applied (Table 1):

These parameters have been determined from preliminary tests aiming at maximizing the convergence speed of the problem (cf. § 5). The value of $p$ must be large enough to ensure optimization algorithm convergence. Since the size of $\Omega(t)$ is growing throughout the optimization process and the convergence of the optimization algorithm requires several iterations, low values of $p$ may limit algorithm performance. Numerical tests also prove that, in the present application case, convergence speed is relatively high and requires mainly high values of the hill-climbing and mutation probabilities compared with most of the values observed in the literature.

\section{Application to a riveted assembly}

The methodology proposed in this paper has been implemented in a design decision-support tool developed in the MATLAB ${ }^{\circledR}$ environment. This system is currently transposed in the post-processing industrial software STREAME used to optimize mechanical assemblies; this software interacts with finite element calculations from other software such as PATRAN ${ }^{\circledR}$-NASTRAN ${ }^{\circledR}$.

Using this software requires two types of expertise: designing expertise and some basic knowledge in optimization. Designing expertise is first used to formalize the observation model of the product behavior, to parameterize the desirability functions and the aggregation functions from design criteria and relative levels of importance. Next, designing expertise is used to define the design objectives and the design variable hierarchy. Finally, optimization expertise is also required to parameterize the genetic algorithm and the exploration sequencing process.

Design expertise and modeling are carried out using the following workflow:

1. The design expert formalizes the observation model with several experts (calculation, aerodynamics, materials, etc.) and through several computational tools. 
2. From customers' requirements, desirability functions are parameterized to define the satisfaction's levels related to every observation variables.

3. The interpretation variables are then aggregated through the aggregation model. The design expert has to specify the aggregation functions, the weight parameters and the design objectives. Several tools are used like Functional Analysis to identify the design objectives or Analytic Hierarchy Process to determine the weighting parameters.

4. Then, the design expert defines the hierarchy between these variables according to his preferences.

5. From experimental observations of the performances of the optimization algorithm (in particular, its convergence speed), the optimization expert can parameterize the sequencing process using the hierarchy specified by the design expert. In particular, the membership functions, the exploration functions and the parameter $p$ (see Section 4.2) are determined.

6. Finally, the optimization expert can parameter the optimization algorithm, according to its knowledge of the algorithm's behavior and the objective function specified by the design expert.

7. At the end of the automatic optimization process, several design solutions are proposed to the designer.

This method can only be employed if:

- The designer is able to specify its preferences related to the physical behavior of the product (desirability curves, aggregation function, weighting parameters).

- The design process is based on a preliminary design solution whose structure (design variable values) is regarded as preferable.

- The design problem isn't too complex (low number of design variables, low CPU time to compute models).

Moreover, to justify such an optimization methodology, the product to design must be relatively recurrent to make necessary model capitalization and justify the model development costs.

In this section, we apply the methodology to the design of a mechanical junction using rivets. The structure of the product is already defined and numerical optimization aims to optimize the number and selection of the type of rivets, rivet line positioning, materials and dimensions of the plates inside the junction. This type of application case proves to be a classic design problem in the aeronautics field and is a major issue since large aircraft contain several million rivets, which is highly penalizing aircraft mass and requires a great deal of manufacturing investment.

\subsection{Observation model}

The fastening junction is made of two identical sheets of aluminum alloy, with parallelepiped shape and linked by a single line of centered and evenly spaced rivets (Fig. 9).

Most approaches used in aeronautics design departments are based on the computation of criteria ensuring the mechanical resistance of the assembly. These criteria are numerical ratios called margins of security (MS) made from the division of an allowable stress and a simulated stress augmented by a security coefficient. Four different MS are taken into account in the model:

$\mathrm{MS}_{k}=\frac{\mathrm{all}_{k}}{1.5 . \mathrm{str}_{k}}-1$ with $k=\left\{\begin{array}{l}1: \text { upset in the sheet } \\ 2: \text { tension stress in the sheet } \\ 3: \text { shears tress in the sheet } \\ 4: \text { shear stress in the fastener }\end{array}\right.$

where $\mathrm{all}_{k}$ is the allowable stress and $\operatorname{str}_{k}$ is the simulated stress.

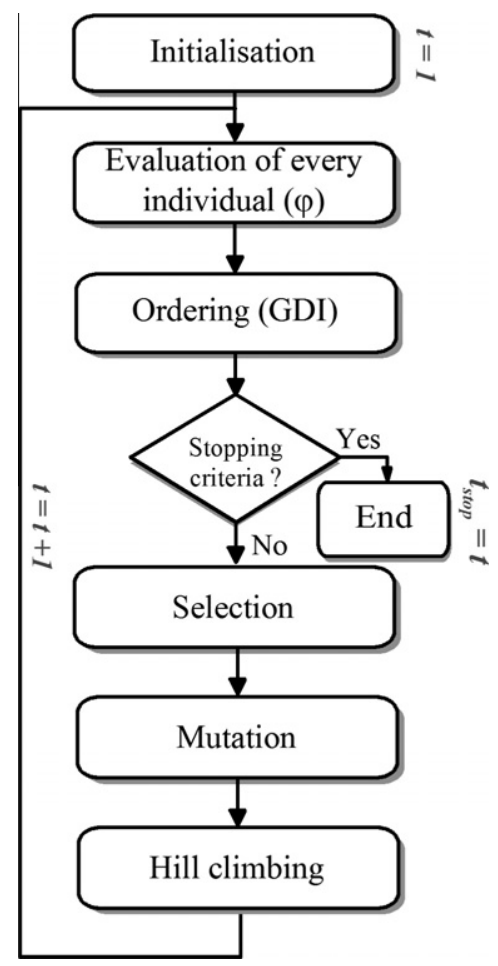

Fig. 8. Genetic algorithm.

Table 1

Parameters used for the method.

$\begin{array}{ll}\text { Genetic algorithm parameters } & \\ \text { Number of individuals per generation } & 100 \\ \text { Cross-over probability } & 85 \% \\ \text { Mutation probability } & 10 \% \\ \text { Hill-climbing probability } & 20 \% \\ \text { GDI }^{\text {stop }} & 0.9 \text { or } 0.95 \\ N_{\text {genMax }} & 1000 \\ \text { Exploration sequencing parameters } & \\ t & \mathrm{~N}_{\text {gen }} \\ p & 20,40 \text { or } 60\end{array}$

To validate the behavior of the assembly, design departments check that the four different values of MS are greater than 0 .

Two other observation variables are added to these structural data, these are the total mass $(M)$ and the unit cost $(P)$ of the assembly.

These six variables constitute the observation variables (vector $\mathrm{Y}$ ) of the model $\mu$. Through the model, their values derive from eight design variables (vector $\mathrm{X}$ ) listed in Table 2 (relations are available in Appendix A.1). In Table 2, the "step" column shows the discretization step for every value domains. The reference numbers for materials and rivets in the table are detailed in Appendix A.2.

Aircraft nacelles are important components which link the jet engine to the mast and the wing. The junction being considered here for design is a sub-assembly of a nacelle. A tensile load of $9000 \mathrm{~N}$ is considered in the following. Fasteners are steel rivets commonly used in aeronautics. The parts consist of thin aluminum sheets and five types of aluminum are commonly used for aeronautical applications. The dimensions of the sheets and the number of rivets of $\mathrm{X}^{0}$ are chosen to respect a traditional know-how rule: the distance between rivets has to be equal to three times the rivet's diameter. Related value domains are centered on the corresponding instantiated variables of $\mathrm{X}^{0}$. 
Table 2

Design variables, observation variables, initial solution.

\begin{tabular}{|c|c|c|c|c|c|}
\hline $\operatorname{order}_{i}$ & Design variables $(\mathrm{X})$ & & Design space $(\bar{\Omega})$ & Step & Init. Sol. $\left(\mathrm{X}^{0}\right)$ \\
\hline 1 & $\mathrm{bl}$ & $\begin{array}{l}\text { Longitudinal distance between the line } \\
\text { of rivets and the sheet edge }(\mathrm{m})\end{array}$ & {$\left[10 \times 10^{-3} ; 50 \times 10^{-3}\right]$} & $2 \times 10^{-3}$ & $30 \times 10^{-3}$ \\
\hline 2 & bt & $\begin{array}{l}\text { Transverse distance between the last rivet } \\
\text { in the line of rivets and the sheet edge ( } \mathrm{m} \text { ) }\end{array}$ & {$\left[10 \times 10^{-3} ; 70 \times 10^{-3}\right]$} & $2 \times 10^{-3}$ & $60 \times 10^{-3}$ \\
\hline 3 & refRiv & Rivet reference $(-)$ & $\{1 ; 2\}$ & - & 1 \\
\hline 4 & Nriv & Number of rivets $(-)$ & {$[2 ; 30]$} & 1 & 15 \\
\hline 5 & $\mathrm{e}$ & Sheet thickness (m) & {$\left[1 \times 10^{-3} ; 10 \times 10^{-3}\right]$} & $1 \times 10^{-3}$ & $8 \times 10^{-3}$ \\
\hline 6 & refMat & Sheet material reference (-) & $\{1 ; 2 ; 3 ; 4 ; 5\}$ & - & 5 \\
\hline 7 & 1 & Sheet width (m) & {$\left[300 \times 10^{-3} ; 500 \times 10^{-3}\right]$} & $2 \times 10^{-3}$ & $400 \times 10^{-3}$ \\
\hline 8 & $\mathrm{~L}$ & Sheet length (m) & {$\left[500 \times 10^{-3} ; 700 \times 10^{-3}\right]$} & $2 \times 10^{-3}$ & $600 \times 10^{-3}$ \\
\hline Observation variables (Y) & & & & & $\left(Y^{0}\right)$ \\
\hline $\mathrm{MS}_{1}$ & & Sheet upset MS (-) & & & 16.85 \\
\hline $\mathrm{MS}_{2}$ & & Sheet tension MS (-) & & & 1.06 \\
\hline $\mathrm{MS}_{3}$ & & Sheet shear MS (-) & & & 9.35 \\
\hline $\mathrm{MS}_{4}$ & & Rivet shear MS (-) & & & 12.06 \\
\hline $\mathrm{M}$ & & Assembly mass (kg) & & & 10.46 \\
\hline $\mathrm{P}$ & & Assembly unit cost $(\$)$ & & & 422.93 \\
\hline
\end{tabular}

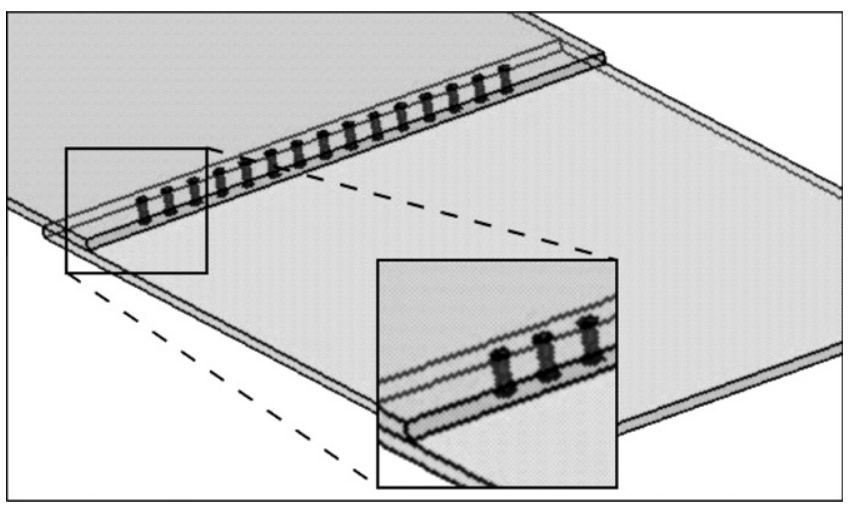

Fig. 9. Simplified riveted assembly (initial solution).

The proposed methodology is based on a preliminary solution (initial solution), which has not yet been optimized since the simulation software STREAME was not able to carry out such an optimization with structural calculations.

\subsection{Interpretation model}

The parameters of the desirability curves corresponding to the criteria of the riveted junction are given in Table 3. While verifying that the safety margins are positive, the aim is to minimize the mass and unit costs. The corresponding accurate limits of the desirability functions are based on the values of the cost related observation variables of $\mathrm{Y}^{0}$. Desirability functions of the safety margins are parameterized to make positive values of the margins preferable; the soft limits are fixed to 5 , given that, for instance, a value of 10 is not really more desirable than a value of 7 .

\subsection{Aggregation model}

In the aggregation model, three design objectives are identified:

- The mechanical strength of the assembly $\left(\mathrm{DOI}_{1}\right)$ : this DOI aggregates the interpretation variables linked to the four security margins (MS).

- The mass of the assembly $\left(\mathrm{DOI}_{2}\right)$ : this DOI depends only on the desirability of the mass; consequently, its value is the value of the desirability of M.
Table 3

Parameters of desirability functions.

\begin{tabular}{|c|c|c|c|c|c|}
\hline & \multirow[t]{2}{*}{$\mathrm{d}\left(\mathrm{y}_{\mathrm{i}}\right)$} & \multicolumn{2}{|c|}{ for $A C_{i}<S L_{i}$} & \multicolumn{2}{|c|}{ for $A C_{i}>S L_{i}$} \\
\hline & & $\mathrm{AC}_{\mathrm{i}}$ & $\mathrm{SL}_{\mathrm{i}}$ & $\mathrm{SL}_{\mathrm{i}}$ & $\mathrm{AC}_{\mathrm{i}}$ \\
\hline MS1 & $\mathrm{d}(\mathrm{MS} 1)$ & 0 & 5 & - & - \\
\hline MS2 & $\mathrm{d}(\mathrm{MS} 2)$ & 0 & 5 & - & - \\
\hline MS3 & d(MS3) & 0 & 5 & - & - \\
\hline MS4 & $\mathrm{d}(\mathrm{MS} 4)$ & 0 & 5 & - & - \\
\hline Mass & $\mathrm{d}(\mathrm{M})$ & - & - & 0 & 12 \\
\hline Unit cost & $d(P)$ & - & - & 10 & 400 \\
\hline
\end{tabular}

- The unit cost of the assembly $\left(\mathrm{DOI}_{3}\right)$ : like $\mathrm{DOI}_{2}$, this DOI corresponds to the desirability of $\mathrm{P}$; consequently, its value is the value of the desirability of $P$.

The four interpretation variables that represent the designer's wishes for the four MS are considered to be of equal importance since no margin is more important than any other. Thus, the weighting parameters of the aggregation function related to $\mathrm{DOI}_{1}$ (Eq. (4)) are equal and whatever $i, \omega_{1}=\omega_{2}=\omega_{3}=\omega_{4}=0.25$.

On the other hand, the aggregation of the three DOI into a single performance value GDI (Eq. (5)) has been weighted according to relative importance levels defined by the designer. The main optimization objective is to minimize the mass of the junction, $\mathrm{DOI}_{2}$ is the most important indicator and its weighting parameter is $v_{2}=0.7$. Next, mechanical strength has to be guaranteed: $v_{1}=0.2$. Finally, the unit cost is the least important design objective: $v_{3}=0.1$.

\subsection{Results and discussion}

To illustrate the proposed approach, several optimizations have been computed and the corresponding results are summed up in Table 4. Two stopping criterion values have been tested corresponding to $\mathrm{GDI}^{\text {stop }}=0.90$ and $\mathrm{GDI}^{\text {stop }}=0.95$. The values of 20,40 or 60 have been used for the three values of the parameter $p$ defining the slopes of the exploration functions. Four hierarchies have also been tested. These hierarchies correspond to four different classifications of the design variables. These computations are completed by two optimization runs performed without hierarchy.

These results show that, using the sequencing exploration method, optimal solutions are found close to the initial reference solution $\left(\mathrm{X}^{0}\right)$. For example, two design variables for solution $\mathrm{X}^{16}$ are equal to the initial variables $(l$ and $L)$ and solution $\mathrm{X}^{21}$ is 
Table 4

Optimal solutions for several sets of parameters.

\begin{tabular}{|c|c|c|c|c|c|c|c|c|c|c|c|c|c|c|c|c|c|c|c|}
\hline \multirow[t]{2}{*}{ Hierarchy } & & \multirow[t]{2}{*}{$\mathrm{p}$} & \multirow[t]{2}{*}{$\mathrm{GDI}^{\text {stop }}$} & \multicolumn{8}{|c|}{ Design variables $\mathrm{X}$} & \multicolumn{6}{|c|}{ Observation variables $\mathrm{Y}$} & \multirow[t]{2}{*}{ GDI } & \multirow[t]{2}{*}{ Sol. } \\
\hline & & & & bl & bt & refRiv & Nriv & $\mathrm{e}$ & refMat & 1 & $\mathrm{~L}$ & $\mathrm{MS}_{1}$ & $\mathrm{MS}_{2}$ & $\mathrm{MS}_{3}$ & $\mathrm{MS}_{4}$ & $\mathrm{M}$ & $\mathrm{P}$ & & \\
\hline & & 20 & 0.90 & 0.05 & 0.01 & 2 & 5 & 0.003 & 2 & 0.4 & 0.6 & 3.77 & 6.39 & 11.99 & 16.3 & 4.12 & 166.59 & 0.93 & $\mathrm{X}^{1}$ \\
\hline 1. bl & 5. e & & 0.95 & 0.05 & 0.01 & 2 & 5 & 0.003 & 2 & 0.3 & 0.59 & 3.77 & 4.16 & 11.98 & 16.3 & 2.7 & 124.87 & 0.95 & $\mathrm{X}^{2}$ \\
\hline 2. bt & 6. refMat & 40 & 0.90 & 0.05 & 0.01 & 2 & 5 & 0.003 & 2 & 0.4 & 0.6 & 3.77 & 6.39 & 11.98 & 16.3 & 4.12 & 166.59 & 0.92 & $\mathrm{X}^{3}$ \\
\hline 3. refRiv & 7.1 & & 0.95 & 0.05 & 0.01 & 2 & 5 & 0.003 & 2 & 0.3 & 0.6 & 3.77 & 4.16 & 11.98 & 16.3 & 3.11 & 137.0 & 0.95 & $\mathrm{X}^{4}$ \\
\hline \multirow[t]{3}{*}{ 4. Nriv } & 8. $\mathrm{L}$ & 60 & 0.90 & 0.05 & 0.01 & 2 & 5 & 0.003 & 2 & 0.4 & 0.6 & 3.77 & 6.39 & 11.98 & 16.3 & 4.12 & 166.59 & 0.92 & $X^{5}$ \\
\hline & & & 0.95 & 0.05 & 0.012 & 2 & 5 & 0.003 & 2 & 0.3 & 0.52 & 3.77 & 4.07 & 11.98 & 16.3 & 2.73 & 125.76 & 0.95 & $\mathrm{X}^{6}$ \\
\hline & & 20 & 0.90 & 0.05 & 0.06 & 2 & 5 & 0.003 & 2 & 0.4 & 0.6 & 3.77 & 4.16 & 11.98 & 16.3 & 4.12 & 166.59 & 0.92 & $\mathrm{X}^{7}$ \\
\hline 1. refMat & 5. e & & 0.95 & 0.05 & 0.01 & 2 & 5 & 0.003 & 2 & 0.3 & 0.55 & 3.77 & 4.16 & 11.98 & 16.3 & 2.84 & 129.02 & 0.95 & $\mathrm{X}^{8}$ \\
\hline 2. Nriv & 6. bt & 40 & 0.90 & 0.048 & 0.06 & 2 & 5 & 0.003 & 2 & 0.4 & 0.6 & 3.77 & 4.16 & 11.48 & 16.3 & 4.12 & 166.59 & 0.92 & $\mathrm{X}^{9}$ \\
\hline 3. refRiv & 7.1 & & 0.95 & 0.048 & 0.01 & 2 & 5 & 0.003 & 2 & 0.3 & 0.54 & 3.77 & 4.16 & 11.48 & 16.3 & 2.89 & 128.72 & 0.95 & $\mathrm{X}^{10}$ \\
\hline \multirow[t]{3}{*}{ 4. bl } & 8. $\mathrm{L}$ & 60 & 0.90 & 0.05 & 0.06 & 2 & 4 & 0.003 & 2 & 0.4 & 0.6 & 2.8 & 5.72 & 11.98 & 12.84 & 4.11 & 160.94 & 0.91 & $\mathrm{X}^{11}$ \\
\hline & & & 0.95 & 0.05 & 0.01 & 2 & 5 & 0.003 & 2 & 0.3 & 0.55 & 3.77 & 4.16 & 11.98 & 16.3 & 2.84 & 129.02 & 0.95 & $\mathrm{X}^{12}$ \\
\hline & & 20 & 0.90 & 0.048 & 0.012 & 2 & 4 & 0.003 & 2 & 0.4 & 0.6 & 2.81 & 8.39 & 11.48 & 12.84 & 4.11 & 160.94 & 0.91 & $\mathrm{X}^{13}$ \\
\hline 1. e & 5. bl & & 0.95 & 0.05 & 0.01 & 2 & 5 & 0.003 & 2 & 0.3 & 0.53 & 3.77 & 4.16 & 11.98 & 16.3 & 2.78 & 127.24 & 0.95 & $\mathrm{X}^{14}$ \\
\hline 2. Nriv & 6. refMat & 40 & 0.90 & 0.05 & 0.01 & 2 & 5 & 0.003 & 2 & 0.4 & 0.6 & 3.77 & 6.39 & 11.98 & 16.3 & 4.12 & 166.59 & 0.92 & $X^{15}$ \\
\hline 3. refRiv & 7.1 & & 0.95 & 0.05 & 0.01 & 2 & 5 & 0.003 & 2 & 0.302 & 0.542 & 3.77 & 4.21 & 11.98 & 16.3 & 2.84 & 128.96 & 0.95 & $\mathrm{X}^{16}$ \\
\hline \multirow[t]{3}{*}{ 4. bt } & 8. $\mathrm{L}$ & 60 & 0.90 & 0.05 & 0.01 & 2 & 5 & 0.003 & 2 & 0.4 & 0.6 & 3.77 & 6.39 & 11.98 & 16.3 & 4.12 & 166.59 & 0.92 & $\mathrm{X}^{17}$ \\
\hline & & & 0.95 & 0.05 & 0.01 & 2 & 5 & 0.003 & 2 & 0.3 & 0.538 & 3.77 & 4.16 & 11.98 & 16.3 & 2.8 & 127.83 & 0.95 & $\mathrm{X}^{18}$ \\
\hline & & 20 & 0.90 & 0.05 & 0.01 & 1 & 11 & 0.003 & 2 & 0.5 & 0.5 & 4.26 & 3.32 & 11.83 & 8.58 & 4.23 & 188.92 & 0.91 & $X^{19}$ \\
\hline 1. bl & 5. $\mathrm{L}$ & & 0.95 & 0.05 & 0.01 & 2 & 6 & 0.003 & 2 & 0.324 & 0.5 & 4.72 & 3.57 & 11.98 & 19.77 & 2.82 & 133.78 & 0.95 & $X^{20}$ \\
\hline 2.1 & 6. refRiv & 40 & 0.90 & 0.05 & 0.01 & 1 & 11 & 0.003 & 2 & 0.496 & 0.5 & 4.26 & 3.28 & 11.83 & 8.58 & 4.2 & 187.93 & 0.91 & $\mathrm{X}^{21}$ \\
\hline 3. e & 7. refMat & & 0.95 & 0.05 & 0.01 & 2 & 6 & 0.003 & 2 & 0.362 & 0.5 & 4.72 & 4.27 & 11.98 & 19.77 & 3.14 & 143.15 & 0.95 & $\mathrm{X}^{22}$ \\
\hline \multirow[t]{2}{*}{ 4. bt } & 8. Nriv & 60 & 0.90 & 0.05 & 0.01 & 1 & 11 & 0.003 & 2 & 0.492 & 0.5 & 4.26 & 3.24 & 11.83 & 8.58 & 4.17 & 186.95 & 0.91 & $\mathrm{X}^{23}$ \\
\hline & & & 0.95 & 0.05 & 0.01 & 2 & 5 & 0.003 & 2 & 0.304 & 0.5 & 3.77 & 4.25 & 11.98 & 16.3 & 2.64 & 123.2 & 0.95 & $\mathrm{X}^{24}$ \\
\hline \multirow{2}{*}{ Without hierarchy } & & & 0.90 & 0.05 & 0.018 & 2 & 4 & 0.005 & 2 & 0.304 & 0.51 & 5.35 & 9.64 & 20.64 & 12.84 & 4.41 & 170.01 & 0.92 & $\mathrm{X}^{25}$ \\
\hline & & & 0.95 & 0.044 & 0.012 & 2 & 5 & 0.003 & 2 & 0.342 & 0.512 & 3.77 & 5.01 & 10.46 & 16.3 & 3.03 & 134.59 & 0.95 & $X^{26}$ \\
\hline Initial solution & & & & 0.03 & 0.06 & 1 & 15 & 0.008 & 5 & 0.4 & 0.6 & 16.85 & 1.06 & 9.35 & 12.06 & 10.46 & 422.93 & 0.09 & $\mathrm{X}^{0}$ \\
\hline
\end{tabular}



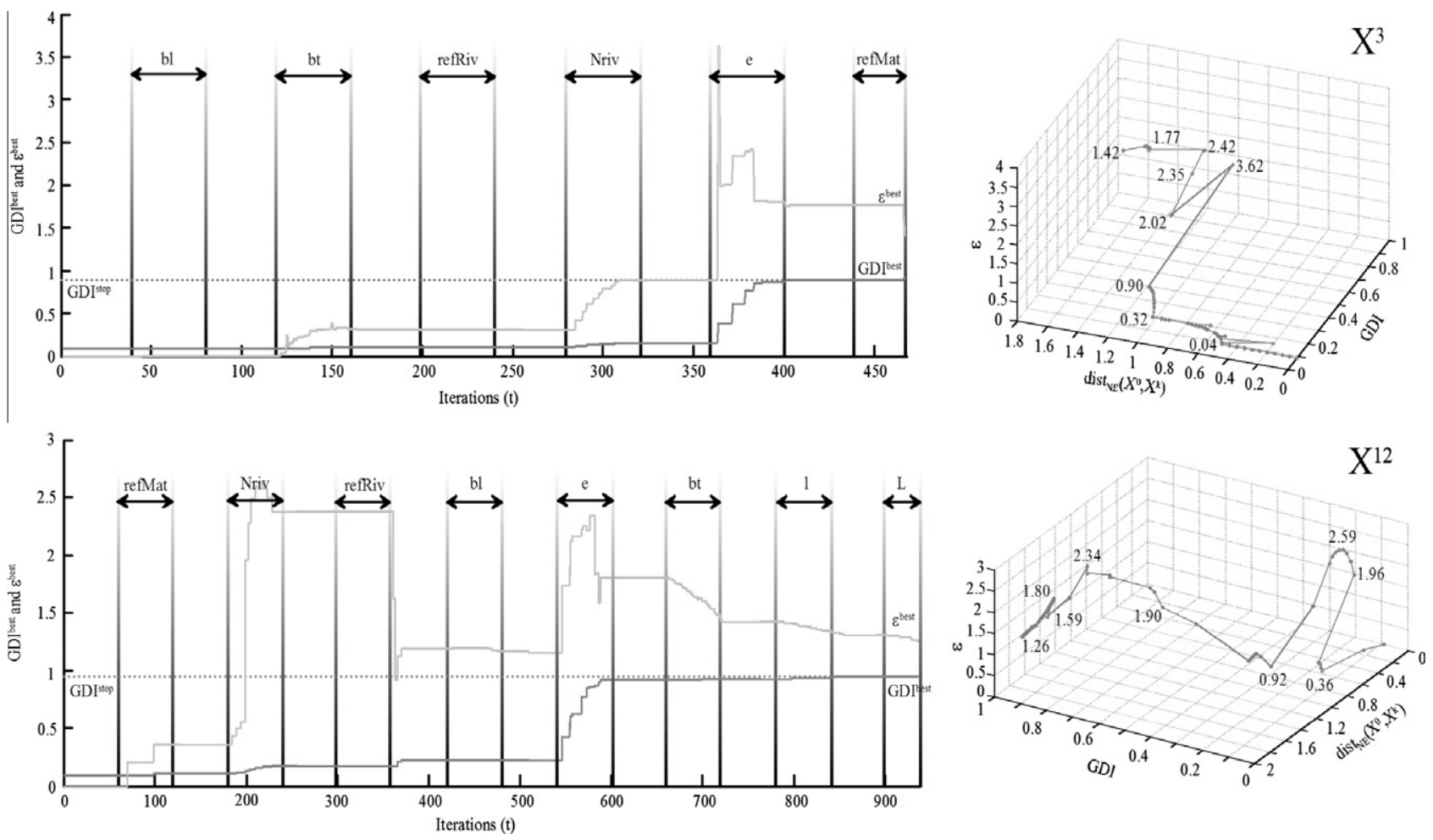

Fig. 10. Best performances and the corresponding arc-elasticity, through two design variables hierarchies.

Table 5

Best design solution according to the arc-elasticity notion.

\begin{tabular}{|c|c|c|c|c|c|c|c|c|c|c|c|c|c|c|c|c|}
\hline & bl & bt & refRiv & Nriv & e & refMat & 1 & $\mathrm{~L}$ & $\mathrm{MS}_{1}$ & $\mathrm{MS}_{2}$ & $\mathrm{MS}_{3}$ & $\mathrm{MS}_{4}$ & M & $\mathrm{P}$ & GDI & $\varepsilon$ \\
\hline $\mathrm{X}_{\varepsilon}^{5}$ & 0.05 & 0.058 & 1 & 7 & 0.005 & 5 & 0.4 & 0.6 & 4.2 & 2.31 & 9.69 & 5.09 & 6.54 & 261.97 & 0.73 & 4.02 \\
\hline $\mathrm{X}^{5}$ & 0.05 & 0.01 & 2 & 5 & 0.003 & 2 & 0.4 & 0.6 & 3.77 & 6.39 & 11.98 & 16.3 & 4.12 & 166.59 & 0.92 & 1.42 \\
\hline $\mathrm{X}^{0}$ & 0.03 & 0.06 & 1 & 15 & 0.008 & 5 & 0.4 & 0.6 & 16.85 & 1.06 & 9.35 & 12.06 & 10.46 & 422.93 & 0.09 & 0 \\
\hline
\end{tabular}

Table 6

Nomenclature of the analytical model.

\begin{tabular}{|c|c|c|c|}
\hline Variable & Description & Unit & \\
\hline \multirow[t]{5}{*}{ refMat } & $\mathrm{Rp}_{0.2}$ & Elastic limit under tension & $\mathrm{Pa}$ \\
\hline & $\mathrm{Rm}$ & Max stress under shear & $\mathrm{Pa}$ \\
\hline & Cupset & Max stress under upset & $\mathrm{Pa}$ \\
\hline & $\rho_{\text {mat }}$ & Volume mass of material & $\mathrm{kg} / \mathrm{m}^{3}$ \\
\hline & Pvol $_{\text {mat }}$ & Volume cost of material & $\$ / \mathrm{m}^{3}$ \\
\hline bt & Transverse distance between last rivet and edge of sheet & $\mathrm{m}$ & \\
\hline bl & Longitudinal distance between line of rivets and edge of sheet & $\mathrm{m}$ & \\
\hline \multirow[t]{4}{*}{ refRiv } & 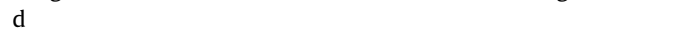 & Diameter of rivet & $\mathrm{m}$ \\
\hline & $\mathrm{Cmax}_{\text {shear }}$ & Max stress under shear & $\mathrm{Pa}$ \\
\hline & $\mathrm{M}_{\text {rivet }}$ & Mass of rivet & $\mathrm{kg}$ \\
\hline & $P_{\text {rivet }}$ & Cost of rivet & $\$$ \\
\hline e & Thickness of sheet & $\mathrm{m}$ & \\
\hline Nriv & Number of rivets & - & \\
\hline Fx & Stress according to $\mathrm{x}$ & $\mathrm{N}$ & \\
\hline $\mathrm{L}$ & Length of sheetws & $\mathrm{m}$ & \\
\hline 1 & Width of sheet & $\mathrm{m}$ & \\
\hline$P_{\text {drilling }}$ & Unit cost of drilling sheet & $\$$ & \\
\hline $\mathrm{P}_{\mathrm{asm} \text { sheet }}$ & Unit cost of handling sheet for assembly & $\$$ & \\
\hline $\mathrm{P}_{\text {asm rivet }}$ & Unit cost of riveting & $\$$ & \\
\hline
\end{tabular}

constituted by 11 fasteners, whereas the initial solution is constituted of 15 fasteners. These design variables are slightly modified because of their ranks in the hierarchy; they are ranked at the end of the hierarchy, and are modified if the stopping criterion is not yet reached.
In other words, this criterion acts on the distance between the optimal solution and the initial one. For example, in the first hierarchy, with the same stopping criterion $\mathrm{GDI}^{\text {stop }}=0.90$, optimal solutions are obtained without modifying the two last design variables in the hierarchy $\left(X^{5}\right)$, whereas in a traditional optimization 
process, an optimal solution modifies all the design variables $\left(\mathrm{X}^{25}\right)$. With the solution $\mathrm{X}^{6}$ and a higher stopping criterion, all the design variables are also modified. For a lower stopping criterion, the number of modified variables would obviously be lower.

On the other hand, the value of the parameter $p$ has a low importance since it has no impact on the optimal solution considering the three values tested. For example, solutions $\mathrm{X}^{1}, \mathrm{X}^{3}$ and $\mathrm{X}^{5}$ are identical and $\mathrm{X}^{14}, \mathrm{X}^{16}, \mathrm{X}^{18}$ are very similar (only the last variable " $L$ " is a little different, due to the behavior of the stochastic algorithm). However, the value of $p$ must be high enough to minimize its influence on the algorithm performance, which requires numerical tests and expertise on the subject.

To conclude, the optimal solution depends on the stopping criterion and the hierarchy specified by the designer. If the stopping criterion is low, only the first design variables in the hierarchy are modified.

In the following paragraphs, the parameters' values employed are:

- First hierarchy in the Table 4.

- $p=60$.

- $\mathrm{GDI}^{\text {stop }}=0.90$.

Fig. 10 shows the evolution of the performance of the best candidate solution GDI ${ }^{\text {best }}$ for every iteration of the genetic algorithm, and the evolution of the arc-elasticity $\varepsilon^{\text {best }}$ for this solution. The processing zone of a design variable is the area with a value t corresponding to the decreasing portion of its exploration function $\alpha_{i}$ (cf. $\S 4.2$ ). These processing zones are indicated by arrows in Fig. 10. It details the convergence for solutions $X^{3}$ and $X^{12}$ (Table 4).

This figure shows the sequencing of the design space exploration and the impact of processing every design variable on the evolution of the GDI of the solutions, starting from the initial solution $\mathrm{X}^{0}$ (for $t<40$ ). This impact may vary, depending on the variables. The impact of the design variable "Nriv" on the evolution of performance is different for solutions $\mathrm{X}^{3}$ and $\mathrm{X}^{12}$. Consequently, every performance improvement is not due solely to a given variable, but to a combination of this variable and the design variables processed previously. Fig. 10 shows that, at the beginning of the optimization, the arc-elasticity of performance $\varepsilon^{\text {best }}$ increases with performance, which proves that the increase in performance remains interesting compared to the increase in the distance between the candidate solution and the initial one. The arc-elasticity then decreases, which indicates that this distance becomes too great to be compensated by performance improvements. This evolution in the arc-elasticity values is shown in the threedimensional diagrams in Fig. 10. These diagrams illustrate the path of the performance maximization on the arc-elasticity mapping (Fig. 5).

This evolution in the arc-elasticity value is explained by the maximization of the performance, and not by the maximization of the arc-elasticity itself. Finally, we use the arc-elasticity value to rank all the candidate solutions evaluated during an optimization run, as a post-processing ranking. The run achieving solution $\mathrm{X}^{5}$ is thus post-processed (parameters are given in Table 4). Table 5 shows the characteristics of the candidate solution maximizing the arc-elasticity value - denoted as $\mathrm{X}_{\varepsilon}^{5}$.

The arc-elasticity-oriented optimal solution $\mathrm{X}_{\varepsilon}^{5}$ has an arc-elasticity value of 4.02 . It is observed that this solution is very similar to the initial solution. Four design variable values are equal to the design variables of solution $X^{0}$ ("refRiv", "refMat", "l" and " $L$ ") and the values of the "bt" variable are very close $(0.058 \approx 0.06)$ to the corresponding value of solution $\mathrm{X}^{0}$. Most of the observation variables of $\mathrm{X}_{\varepsilon}^{5}$ have a better value than $\mathrm{X}^{0}$. The values of $\mathrm{MS}_{2}$ and $\mathrm{MS}_{3}$ are higher, and the values of $M$ and $P$ are lower, which significantly improves the global performance of the design solution.
The arc-elasticity notion performs an efficient trade-off between the performance gain and the cost associated with transforming the initial reference solution. Obviously, the resulting arc-elasticity-oriented optimal solution $\mathrm{X}_{\varepsilon}^{5}$ has a lower performance than the performance-oriented optimal solution $\mathrm{X}^{5}$.

\section{Conclusion}

In this paper, an optimization methodology is proposed to deal with the redesign problems encountered by design departments, starting from an existing design solution. It is based on the Observation-Interpretation-Aggregation modeling approach, which combines models of product physical behavior and designer's preferences. These models qualify the candidate solutions through a single performance variable. This performance value is used to compute arc-elasticity, which takes into account the initial reference solution and the candidate solution. Arc-elasticity is used as a trade-off indicator between performance improvement (maximization) and increase in distance (minimization). Using a sequencing exploration of the design space, the designers are able to prioritize design variables, and thus direct optimization.

The proposed method has been illustrated through the design of a riveted junction used in aeronautics. The contribution of the optimization method greatly depends on the performance of the initial reference solution $\mathrm{X}^{0}$.

This method therefore concerns industrial redesign applications where the designer want to make an existing design configuration evolve towards new design applications or specifications. For new applications, pre-existing solutions are often relatively inefficient and must be improved. It also concerns industrial redesign applications where the designers want to optimize or prove the optimal characteristics of an existing configuration. Our methodology is currently applied to complex aeronautic examples, to evaluate its contribution to a real design process.

The specific feature of the method presented in this paper is the combination of exploration sequencing and arc-elasticity optimization. With this natural and intuitive combination, the hierarchy of the design variables is easily performed. However, such a sequencing methodology is highly time-consuming and depends on the optimization of algorithm convergence and design problem complexity (shape of the objective function). This requires a high knowledge level of the algorithm's behavior taking into account the objective function.

Consequently, it would be interesting to propose a model of the closeness between the candidate solution and the initial solution, replacing the dimensionless Euclidean distance and integrating the design variables hierarchy. This formalization would be a new model of the generalized cost integrating other designer's preferences. Finally, in future work, the authors plan to optimize the arc-elasticity value directly, instead of using it through postprocessing.

\section{Acknowledgments}

This work is a part of workpackage 300 in the AXSPAD project. Several partners are contributing to this project: Altep Ingenieries, AXS Analyse de structure and AIRCELLE groupe SAFRAN. In particular, the authors would like to thank Florent Braure, Guillaume Ruiz and Nicolas Gehin.

\section{Appendix A. Appendices}

\section{A.1. Nomenclature}

(see Table 6) 
Table 7

Materials references.

\begin{tabular}{|c|c|c|c|c|c|c|}
\hline refMat & Name & $\mathrm{Rp}_{0.2}(\mathrm{~Pa})$ & $\mathrm{Rm}(\mathrm{Pa})$ & Cupset (Pa) & $\rho_{\text {mat }}\left(\mathrm{kg} / \mathrm{m}^{3}\right)$ & $\operatorname{Pvol}_{\text {mat }}\left(\$ / \mathrm{m}^{3}\right)$ \\
\hline 1 & 2024-T4 & $320 \times 10^{6}$ & $285 \times 10^{6}$ & $500 \times 10^{6}$ & 2780 & 90,468 \\
\hline 2 & 7075-T6 & $500 \times 10^{6}$ & $570 \times 10^{6}$ & $450 \times 10^{6}$ & 2810 & 82,179 \\
\hline 3 & $5050-0$ & $55 \times 10^{6}$ & $145 \times 10^{6}$ & $300 \times 10^{6}$ & 2690 & 44,065 \\
\hline 4 & 6106-T5 & $230 \times 10^{6}$ & $265 \times 10^{6}$ & $400 \times 10^{6}$ & 2700 & 87,963 \\
\hline 5 & 3004-H26 & $250 \times 10^{6}$ & $285 \times 10^{6}$ & $420 \times 10^{6}$ & 2720 & 88,718 \\
\hline
\end{tabular}

Table 8

Rivets references.

\begin{tabular}{llllll}
\hline refRiv & Name & $d(\mathrm{~m})$ & $\operatorname{Cmax}_{\text {shear }}(\mathrm{Pa})$ & $\mathrm{M}_{\text {rivet }}(\mathrm{kg})$ & $\mathrm{P}_{\text {rivet }}(\$)$ \\
\hline 1 & HSR200-6 & $4.78 \times 10^{-3}$ & $655 \times 10^{6}$ & $4.2 \times 10^{-3}$ & 3 \\
2 & HSR209-12 & $9.53 \times 10^{-3}$ & $655 \times 10^{6}$ & $16.5 \times 10^{-3}$ & 4.5 \\
\hline
\end{tabular}

\section{A.2. Rivets references and materials references}

The values detailed below are used in the application case: they are not real but quite realistic.

(see Tables 7 and 8).

\section{A.3. Details of the observation model}

MS1 $=\frac{\text { Cbearing }}{1.5 . \mathrm{str}_{1}}-1$ with $\operatorname{str}_{1}=\frac{F x / \text { Nriv }}{\text { d.e }}$

Using Eq. (18) the bearing margin of security can be calculated in relation to the behavior of the sheet.

$$
\begin{aligned}
M S 2 & =\frac{R P_{0.2}}{1.5 . s \operatorname{st}_{2}}-1, \text { with } \operatorname{str}_{2}=\frac{F x}{(p t-d) e} \text { and } p t \\
& =\frac{1-2 . b t}{\text { Nriv }-1}
\end{aligned}
$$

The physical behavior under tension is computed by calculating stress in the section of the sheet which has the same width as the inter-rivet interval (pt) as indicated in Eq. (19).

$$
\begin{aligned}
\text { MS3 } & =\frac{R m}{1.5 \cdot \operatorname{str}_{3}}-1, \text { with } \operatorname{str}_{3}=\frac{F x}{2 \cdot l_{c} \cdot e} \text { and } l_{c} \\
& =b l-d / 2 \cdot \sin (50)
\end{aligned}
$$

Eq. (20) verifies the behavior of the sheet under shear.

MS4 $=\frac{\text { Cmax }_{\text {shear }}}{1.5 . s t r_{4}}-1$ with $s t r_{4}=\frac{F x / \text { Nriv }}{\pi d^{2} / 4}$

The fourth MS (Eq. (21)) verifies the behavior of the rivet under shear.

$$
\begin{aligned}
& M=2\left(V_{\text {sheet }}-N r i v . V_{\text {rivet }}\right) \rho_{\text {mat }}+N r i v . M_{\text {rivet }} \\
& \text { with } V_{\text {sheet }}=\text { L.l.e } \text { and } V_{\text {rivet }}=\pi d^{2} / 4 .^{e}
\end{aligned}
$$

The total mass of the assembly is expressed in Eq. (22).

$$
\begin{aligned}
P= & {\left[\text { Nriv } \cdot P_{\text {rivet }}+2\left(V_{\text {sheet }} \cdot P \text { vol } l_{\text {mat }}\right)\right]+\left[\text { Nriv } \cdot P_{\text {drilling }}\right] } \\
& +\left[2 \cdot P_{\text {asm sheet }}+\text { Nriv } \cdot P_{\text {asm rivet }}\right]
\end{aligned}
$$

Cost is calculated using three terms (Eq. (23)). The first considers only the purchase price of the parts via the unit cost of the rivet and the volume cost of the materials used for the sheets. The second term proposes a cost for each drilling, multiplied by the number of rivets. The third term proposes a cost for assembling the parts, which varies according to the number of rivets.

\section{A.4. Details of the interpretation model}

Two types of functions are used in the interpretation model. These curves are defined by Eq. (24):

$d\left(y_{i}\right)=\exp \left(-\exp \left(\delta_{i}+\lambda_{i} \cdot y_{i}\right)\right)$

With, for an increasing function (Eq. (25)):

if $A C_{i}>S L_{i},\left\{\begin{array}{l}\lambda_{i}=\frac{\ln \left(\ln \left(d_{i}^{A C_{i}}\right) / \ln \left(d_{i}^{S L_{i}}\right)\right)}{A C_{i}-S L_{i}} \\ \delta_{i}=\ln \left(-\ln \left(d_{i}^{S L_{i}}\right)\right)-\lambda_{i} \cdot S L_{i}\end{array}\right.$

And for a decreasing function (Eq. (26)):

if $A C_{i}<S L_{i},\left\{\begin{array}{l}\lambda_{i}=\frac{\ln \left(\ln \left(d_{i}^{S L_{i}}\right) / \ln \left(d_{i}^{A C_{i}}\right)\right)}{S L_{i}-A C_{i}} \\ \delta_{i}=\ln \left(-\ln \left(d_{i}^{S L_{i}}\right)\right)-\lambda_{i} . S L_{i}\end{array}\right.$

\section{References}

[1] K. Saridakis, A. Dentsoras, Soft computing in engineering design - A review, Advanced Engineering Informatics 22 (2008) 202-221.

[2] N. Kaya, Machining fixture locating and clamping position optimization using genetic algorithms, Computers in Industry 57 (2006) 112-120.

[3] M. Martz, W. Neu, Multi-Objective Optimization of an Autonomous Underwater Vehicle, Marine Technology Society Journal 43 (2009) 48-60.

[4] V. Alexandre, W. Gasparski, The Roots of Praxiology: French Action Theory from Bourdeau and Epinas to Present Days, Transaction Publishers, 2000.

[5] D.G. Ullman, Trade Studies with Uncertain Information, in Proceeding of the Sixteenth Annual International Symposium of the International Council On Systems Engineering (INCOSE), 2006.

[6] O.B. Augusto, S. Rabeau, P. Dépincé, F. Bennis, Multi-objective genetic algorithms: A way to improve the convergence rate, Engineering Applications of Artificial Intelligence 19 (2006) 501-510.

[7] D. Jones, S. Mirrazavi, M. Tamiz, Multi-objective meta-heuristics: an overview of the current state-of-the-art, European Journal of Operational Research 137 (2002) 1-9.

[8] C. Romero, M. Tamiz, D.F. Jones, Goal programming, compromise programming and reference point method formulations: linkages and utility interpretations, The Journal of the Operational Research Society 49 (1998) 986-991.

[9] T. Tomiyama, P. Gu, Y. Jin, D. Lutters, C. Kind, F. Kimura, Design methodologies: Industrial and educational applications, CIRP Annals - Manufacturing Technology 58 (2009) 543-565.

[10] J.V. Neumann, O. Morgenstern, Theory of Games and Economic Behaviour, Science editions., J. Wiley, 1944.

[11] M. Marston, F. Mistree, An implementation of expected utility theory in decision based design, in: ASME Design Engineering and Technical Conferences, Atlanta, GA, DETC98/DTM-5670, 1998.

[12] K.L. Wood, E.K. Antonsson, Computations with imprecise parameters in engineering design: background and theory, Journal of Mechanisms, Transmissions, and Automation in Design 111 (1989) 619-625.

[13] K.N. Otto, E.K. Antonsson, Trade-off strategies in engineering design, Research in Engineering Design 3 (1991) 87-103.

[14] M. Scott, E. Antonsson, Aggregation functions for engineering design tradeoffs, Fuzzy Sets and Systems 99 (1998) 253-264.

[15] T. Quirante, Y. Ledoux, P. Sebastian, Design optimization of two-stage flash evaporator: a trade-off between design objectives, in: Research in Interactive Design, Springer Paris Berlin Heidelberg New York, Bordeaux, 2010.

[16] A. Collignan, P. Sebastian, J. Pailhes, Y. Ledoux, Qualification of solutions in mechanical design through the concept of elasticity, in: Research in Interactive Design, Springer Paris Berlin Heidelberg New York, Bordeaux, 2010.

[17] E.C. Harrington, The desirability function, Industrial Quality Control 21 (1965) 494-498.

[18] R. Derringer, A balancing act: optimizing a product's properties, Quality Progress 27 (1994) 51-58.

[19] R. Derringer, R. Suich, Simultaneous optimization of several response variables, Journal of Quality Technology 12 (1980) 214-219. 
[20] A. Marshall, The elasticity of wants, in: Principles of Economics, London: Macmillan and Co., Ltd., 1890.

[21] R.G.D. Allen, A.P. Lerner, The concept of arc elasticity of demand, The Review of Economic Studies 1 (1934) 226-230.

[22] M. Scott, Formalizing Negotiation in Engineering Design, PhD Thesis, California Institute of Technology, 1999.

[23] S.H.R. Pasandideh, S.T.A. Niaki, Multi-response simulation optimization using genetic algorithm within desirability function framework, Applied Mathematics and Computation 175 (2006) 366-382.

[24] J. Kros, C. Mastrangelo, Comparing methods for the multi-response design problem, Quality and Reliability Engineering International 17 (2001) 323-331.

[25] R. Chenouard, L. Granvilliers, P. Sebastian, Search heuristics for constraintaided embodiment design, AI EDAM-Artificial Intelligent for Engineering Design Analysis and Manufacturing 23 (2009) 175-195.

[26] M. Scott, E.K. Antonsson, Aggregation Functions For Engineering Design TradeOffs, 9th International Conference on Design Theory and, Methodology 2 (1995) 389-396.

[27] A. Sankarasubramanian, R. Vogel, J. Limbrunner, Climate elasticity of streamflow in the United States, Water Resources Research 37 (2001) 17711781.

[28] E.P. Fenichel, J.I. Tsao, M.L. Jones, Modeling fish health to inform research and management : Renibacterium salmoninarum dynamics in Lake Michigan, Ecological Applications 19 (2009) 747-760.
[29] A.A. Rassafi, M. Vaziri, Application of data envelopment analysis in identifying milestones for passenger and freight transportation sustainability, Scientia Iranica 12 (2005) 426-436.

[30] X. Drèze, F. Zufryden, Measurement of online visibility and its impact on internet traffic, Journal of Interactive Marketing 18 (2004) 20-37.

[31] A. Collignan, Méthode d'optimisation et d'aide à la décision en conception mécanique: Application à une structure aéronautique, $\mathrm{PhD}$ thesis, Université Bordeaux 1, 2011.

[32] P. Sebastian, Y. Ledoux, A. Collignan, J. Pailhes, Linking objective and subjective modeling in engineering design through arc-elastic dominance, Expert Systems with Applications. to be published (DOI: 10.1016/j.eswa.2012.01. 079), (2012).

[33] C. Sung-Hyuk, Comprehensive survey on distance/similarity measures between probability density functions, International Journal of Mathematical Models and Methods in Applied Sciences 1 (2007) 300-307.

[34] E. Elbeltagi, T. Hegazy, D. Grierson, Comparison among five evolutionary-based optimization algorithms, Advanced Engineering Informatics 19 (2005) 43-53.

[35] D.E. Goldberg, Genetic Algorithms in Search, Optimization, and Machine Learning, 1st ed., Addison-Wesley Professional, 1989.

[36] A. Javadi, R. Farmani, T. Tan, A hybrid intelligent genetic algorithm, Advanced Engineering Informatics 19 (2005) 255-262. 